\title{
咔唑及其衍生物合成方法研究进展
}

\author{
房旭涁 $a, b$ 房雷*,a,b苟少华*,a,b \\ ( ${ }^{a}$ 东南大学药物研究中心 南京 211189) \\ ( ${ }^{b}$ 江苏省生物药物高技术研究重点实验室 南京 211189$)$
}

\begin{abstract}
摘要 味唑及其衍生物是一类重要的含氮芳杂环化合物, 具有许多独特的理化性质及生物活性, 分析、归纳、总结了 近 5 年来发表的文献, 选取具有代表性的实例, 并按照中间体的不同将合成方法分为四类, 分别讨论了咔唑及其衍生 物合成方法的进展情况，对咔唑及其衍生物成环的新方法给予重点关注.

关键词 咔唑; 衍生物; 合成方法; 研究进展
\end{abstract}

\section{Progresses in Synthetic Methods of Carbazole and Its Derivatives}

\author{
Fang, Xubin ${ }^{a, b}$ Fang, Lei* ${ }^{*, a, b}$ Gou, Shaohua ${ }^{*, a, b}$ \\ ( ${ }^{a}$ Pharmaceutical Research Center, Southeast University, Nanjing 211189) \\ ( ${ }^{b}$ Jiangsu Province Hi-Tech Laboratory for Bio-medical Research, Southeast University, Nanjing 211189)
}

\begin{abstract}
Carbazole and its derivatives are a class of important nitrogen-containing heterocyclic compounds possessing various unique properties and biological activity. Herein the current progress of their synthetic strategies reported in the past 5 years is reviewed. The representative examples, which have been divided into four categories according to the usage of different key intermediates, are selected and discussed in detail. Special emphasis is put on the newly developed cyclization reactions which may be useful for the construction of the tricyclic backbone of carbazoles.
\end{abstract}

Keywords carbazole; derivatives; synthetic methods; progress

咔唑是一种含氮的杂环化合物, 是许多精细化学品 的中间体，可用于制作塑料、农药、杀虫剂、医药以及 新型聚合物材料等. 在咔唑分子特定位置引入各种取代 基团或官能团对其进行化学修饰, 可以获得多类结构新 颖的咔唑衍生物，目前已有多个被当作药物或药物中间 体被加以开发, 例如许多的咔唑衍生物显示出良好抗肿 瘤活性和抗惊厥活性, 这也体现了咔唑类化合物在医药 方面的应用潜力 ${ }^{[1 \sim 4]}$. 近年来, 随着研究和生产需求的 不断增长, 对咔唑及其类似物的需求量呈逐年上升的趋 势, 传统方法合成的化合物虽然比较经济, 但是产品单 一, 不能满足多样性需要. 因此, 研究咔唑的合成方法 并使之便于工业化生产具有相当重要的意义.

\section{1 传统方法}

咔唑的传统合成方法主要包括 Graebe-Ullmann 反
应、Bucherer 咔唑合成和 Borsche-Drechsel 环化反应. Graebe-Ulmann 反应, 由氨基二苯胺经重氮化反应, 加 热脱掉氮气得到咔唑, 如 Scheme 1a 所示. 该反应由 Graebe 和 Ullmann ${ }^{[1 \mathrm{a}]}$ 于 1896 年报道, Ullmann 通过该方 法合成了几个咔唑化合物, 但是其他研究人员发现不饱 和基团的存在对该反应会产生不利影响. Preston, Tucker 和 Cameron ${ }^{[2]}$ 首次通过该方法获得了硝基咔唑 (微量)、乙酰基咔唑(22\%)和氧基咔唑(34\%)，但收率都 不是很理想. Bucherer 咔唑合成方法是 Bucherer ${ }^{[3 \mathrm{a}]}$ 于 1904 年报道, 由芳肼与萗酚在亚硫酸氢钠存在下共热 反应合成了苯并咔唑, 如 Scheme 1b 所示. BorscheDrechsel 环化反应是 Drechsel 和 Borsche 分别于 1868 年 和 1904 年报道 ${ }^{[\mathrm{b}, 3 \mathrm{~b}]}$, 以苯肼与环已酮缩合成腙, 后者在 酸性条件下环化生成四氢咔唑, 再经催化脱氢制得咔 ${ }^{\square 从}{ }^{[4]}$, 如 Scheme 1c 所示. 得益于催化脱氢方法的发展, Borsche 方法已经成为一条操作简便、条件温和、成本

\footnotetext{
*E-mail: sgou@seu.edu.cn; lei.fang@seu.edu.cn

Received September 3, 2011; revised January 10, 2012; accepted February 8, 2012.

Project supported by the National Natural Science Foundation of China (No. 81001361) and the Ph.D. Programs Foundation of Ministry of Education of China (No. 20100092120046).

国家自然科学基金(No. 81001361)和教育部博士点基金青年教师(Nos. 20100092120046)资助项目.
} 
低廉、产率高的咔唑合成路线, 具有较高的工业化生产 价值.<smiles>Nc1ccccc1Nc1ccc(NOc2cccc3nnn(-c4ccccc4)c23)cc1</smiles>

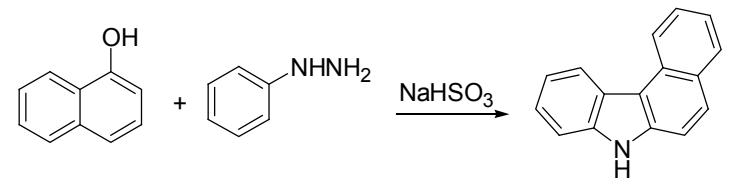<smiles>NNc1ccccc1</smiles>

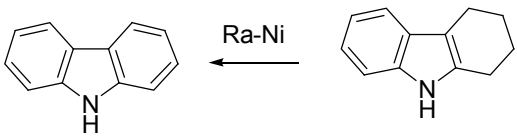

Scheme 1

此外, 工业上也常常采用 2-氨基联苯氧化脱氢或 2,2'一二氨基联苯脱氨制备咔唑 ${ }^{[5]}$, 如 Scheme 2 所示.

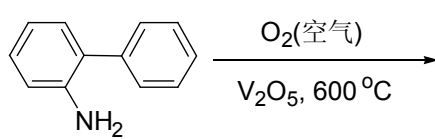<smiles>c1ccc2c(c1)[nH]c1ccccc12</smiles><smiles>Nc1ccccc1-c1ccccc1N</smiles><smiles>N[N+]([NH3+])([O-])[O-]</smiles><smiles>c1ccc2c(c1)[nH]c1ccccc12</smiles>

Scheme 2

\section{2 新方法}

20 世纪最后 10 年中, 大量新颖的贵金属和过渡金 属催化剂被应用于咔唑及其衍生物的合成中, 极大地丰 富了合成手段, 为合成更为复杂的咔唑衍生物奠定了基 础. 如今已不仅仅局限于简单地合成咔唑, 数以万计的 咔唑衍生物通过新的方法被合成出来, 并广泛应用于医 药和染料工业中. 按照中间体的不同, 本文将咔唑及其 衍生物的合成方法分为四类: 联苯、二苯胺、吲哚和其 它.

\section{1 以联苯为中间体的方法}

以联苯为中间体的合成方法得益于 Suzuki-Miyaura 偶联反应的发现。该方法的基本合成策略是利用
Suzuki-Miyaura 偶联反应首先合成带有不同取代基的联 苯中间体，然后利用不同的含氮基团以及使用相应的催 化剂环合得到目标物(Scheme 3). Suzuki-Miyaura 偶联与 Ullmann 等方法相比, 其反应温度通常不超过 $100{ }^{\circ} \mathrm{C}$, 而且在水、醇和醚的混合物体系中即可进行反应，反应 条件及收率上都具有显著优势，有着广阔的应用前景. 例如，咔唑心安(用于高血压)的重要中间体 4-差基咔唑 在合成中依然采用大大过量的铜粉作为催化剂, 高温 $185{ }^{\circ} \mathrm{C}$ 反应，制备联苯收率仅为 $50 \% \sim 60 \%$ ，若采用 $\mathrm{Pd}$ 催化 Suzuki-Miyaura 偶联则收率可提高至 95\% 100\%, 催化剂用量也缩小至 $5 \mathrm{~mol} \%$, 温度降至 $90{ }^{\circ} \mathrm{C}$ 左右, 这 样可以大量节约能源, 减少环境污染, 提高效率.
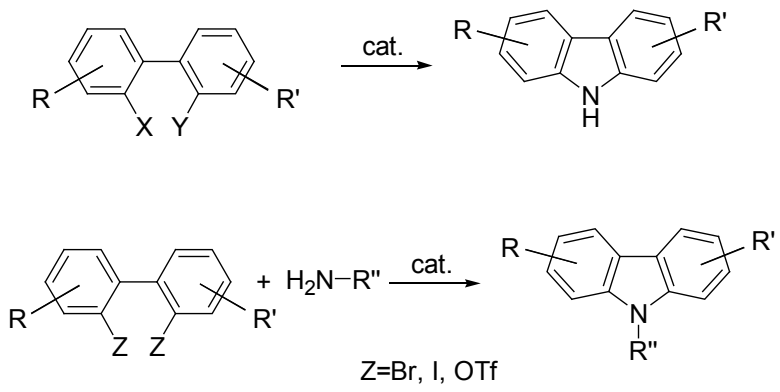

\section{Scheme 3}

在环合步骤中，可以利用成环的基团主要有 $\mathrm{NO}_{2}$, $\mathrm{N}_{3}$, NHR 等, 相应的催化剂见表 1 . 当 $X$ 和 $Y$ 是卤素或 OTf 时，则需要外加氨基化合物，作为咔唑的氮源.

表 $1 \mathrm{X}, \mathrm{Y}, \mathrm{Z}$ 取代基及催化剂 ${ }^{a}$

Table 1 X, Y, Z-substituent and the catalysts

\begin{tabular}{ccclc}
\hline $\mathrm{X}$ & $\mathrm{Y}$ & $\mathrm{Z}$ & \multicolumn{1}{c}{ Cat. } & Yield/\% \\
\hline $\mathrm{NO}_{2}$ & $\mathrm{H}$ & - & $\mathrm{MoO}_{2} \mathrm{Cl}_{2}(\mathrm{dmf})_{2}$ & 87 \\
$\mathrm{~N}_{3}$ & $\mathrm{H}$ & - & $\mathrm{Rh}_{2}\left(\mathrm{O}_{2} \mathrm{CC}_{3} \mathrm{~F}_{7}\right)_{4}$ & 98 \\
$\mathrm{NHR}$ & $\mathrm{H}$ & - & $\mathrm{Pd}(\mathrm{OAc})_{2}$ & 96 \\
- & - & $\mathrm{Br}$ & $\mathrm{Pd}_{2}(\mathrm{dba})_{3}$ & 59 \\
- & - & $\mathrm{I}$ & $\mathrm{CuI}$ & 98 \\
- & - & OTf & $\mathrm{Pd}(\mathrm{dba})_{2}$ & 97 \\
\hline${ }^{a}$ dba $=$ dibenzylideneacetone. & &
\end{tabular}

在各种环合反应中，以硝基为环合基团的应用最为 广泛. Arnáiz 小组 ${ }^{[6]}$ 以各种二取代的硝基联苯为原料, 利用 $\mathrm{MoO}_{2} \mathrm{Cl}_{2}(\mathrm{dmf})_{2}$ 为催化剂在 $\mathrm{PPh}_{3}$ 存在的条件下合成 了一系列的咔唑及咔唑衍生物 1 9, 其收率达 70\% $87 \%$ (Eq. 1), 该方法以 $\mathrm{MoO}_{2} \mathrm{Cl}_{2}(\mathrm{dmf})_{2}$ 为催化剂, 价格 相对低廉，与贵金属催化剂相比具有明显的成本优势, 极具应用价值. 2007 年, Carter 等 ${ }^{[7]}$ 报道了使用 Diels-Alder 反应同样可以获得多取代硝基联苯中间体， 收率达 58\% 78\%，比 Suzuki 偶联方法(22\% 98\%)表 现出更好的稳定性. 以硝基联苯为关键中间体，经 $\mathrm{PPh}_{3}$ 二 二氯苯体系环合，同样可合成一系列咔唑衍生物， 
如 Siamenol 等(Scheme 4).

$$
\text { toluene, } \Delta
$$

1: $\mathrm{R}^{1}=\mathrm{H}, \mathrm{R}^{2}=\mathrm{H} \quad$ 4: $\mathrm{R}^{1}=\mathrm{CHO}, \mathrm{R}^{2}=\mathrm{H} \quad$ 7: $\mathrm{R}^{1}=\mathrm{CO}_{2} \mathrm{Et}, \mathrm{R}^{2}=\mathrm{H}$ 2: $\mathrm{R}^{1}=t$-Bu, $\mathrm{R}^{2}=t$-Bu 5: $\mathrm{R}^{1}=\mathrm{COMe}, \mathrm{R}^{2}=\mathrm{H}$ 8: $\mathrm{R}^{1}=\mathrm{OH}, \mathrm{R}^{2}=\mathrm{H}$ 3: $R^{1}=\mathrm{OMe}, \mathrm{R}^{2}=\mathrm{H} \quad$ 6: $\mathrm{R}^{1}=\mathrm{F}, \mathrm{R}^{2}=\mathrm{H} \quad$ 9: $\mathrm{R}^{1}=\mathrm{CO}_{2} \mathrm{H}, \mathrm{R}^{2}=\mathrm{H}$

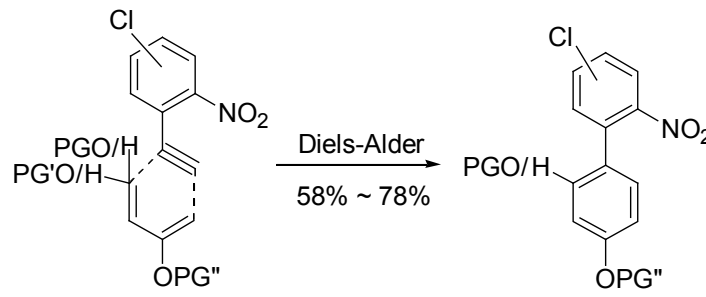

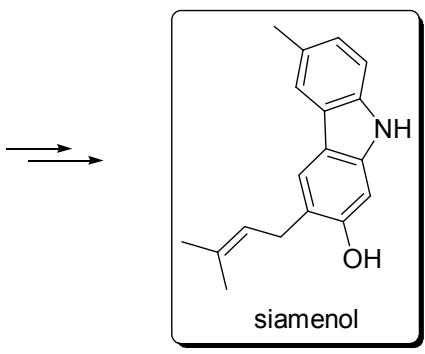

Scheme 4

通常情况下, 硝基联苯的环合反应需要高温长时间 反应(5 12 h)才能反应完全, 2009 年, Creencia 等 ${ }^{[8]}$ 报道, 以硝基联苯为原料, 在 $\mathrm{P}(\mathrm{OEt})_{3}$ 或 $\mathrm{PPh}_{3}$ 存在下, 使用微 波辅助合成了咔唑, 在微波功率为 $200 \mathrm{~W}$ 条件下, 反应 仅 $2 \mathrm{~min}$ 就获得环合产物，收率高达 96\% (Eq. 2).
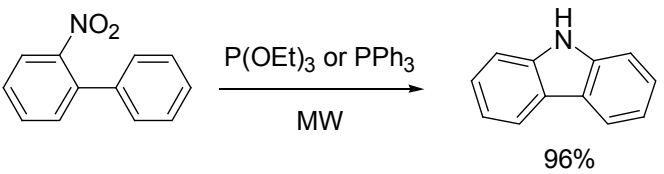

以叠氮基为环合基团同样是合成咔坐的有效手段. 2007 年, Sapi 等 ${ }^{[9]}$ 通过 Suzuki-Miyaura 偶联反应得到叠 氮基联苯, 后者加热释放出氮气, 最终得到咔唑 (Scheme 5). 试验结果显示叠氮联苯上的取代基的不同, 收率上存在很大差异, 波动范围在 $51 \%$ 71\%. 研究发 现, 当联苯中间体一端为苯并吲哚时, 产物具有良好的 选择性, 提示取代基的电性特点对环合位点具有重要影 响. 2009 年, Driver 等 ${ }^{[10]}$ 以叠氮基联苯为原料, 分别以
$\mathrm{Rh}_{2}\left(\mathrm{O}_{2} \mathrm{CC}_{3} \mathrm{~F}_{7}\right)_{4}(5 \mathrm{~mol} \%), 4 \AA \mathrm{MS}(100 \mathrm{wt} \%), \mathrm{PhMe}, 60$ ${ }^{\circ} \mathrm{C}$ 和 $\mathrm{Rh}_{2}\left(\mathrm{O}_{2} \mathrm{CC}_{7} \mathrm{H}_{15}\right)_{4}(5 \mathrm{~mol} \%), 4 \AA \mathrm{MS}(100 \mathrm{wt} \%)$, $\left(\mathrm{CH}_{2} \mathrm{Cl}\right)_{2}, 60{ }^{\circ} \mathrm{C}$ 两个条件合成了 23 个咔唑衍生物, 该方 法收率较高，达到 65\% 98\% (Scheme 6), 提示铑催化 反应具有明显优势. 同时该研究还讨论了环合时的区域 选择性(14：15), 结果显示, 其中 $\mathrm{R}^{\prime}$ 为氯或三氟甲基时 $14: 15>95: 5, \mathrm{R}$ 为氟时比例也高达 $89: 11$, 然而当 $\mathrm{R}$ 为甲基比例仅为 $66: 34$ ，为甲氧基时几乎没有选择性 (Scheme 6, 表 2), 这一发现进一步补充了 Sapi 等的关于 取代基与环合位点关系的理论.
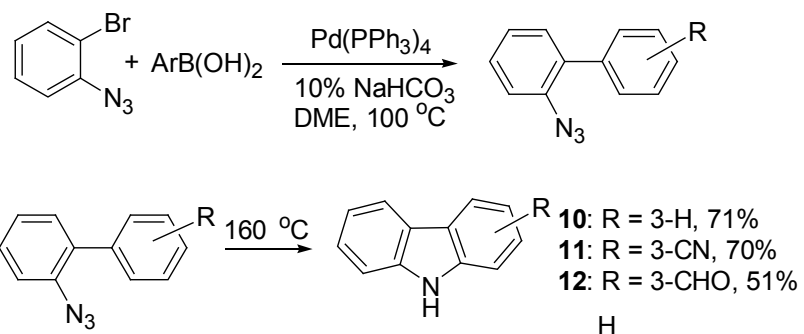<smiles>N#Cc1ccccc1-c1ccc2[nH]ccc2c1</smiles><smiles>c1ccc2c(c1)[nH]c1cc3cc[nH]c3cc12</smiles>

13: $65 \%$

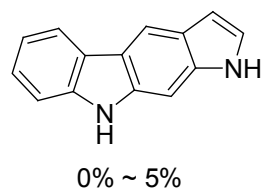

Scheme 5
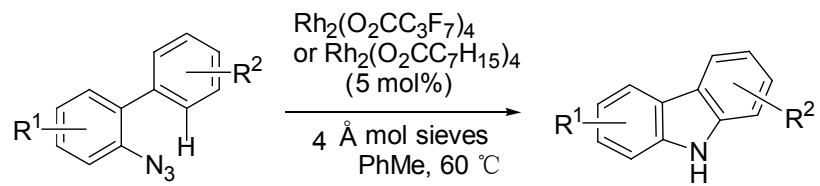<smiles>[R]c1ccc(-c2ccccc2N)cc1[R]</smiles><smiles>[R6]OC(=O)C(C)(F)F</smiles><smiles>[R]c1cc2[nH]c3ccccc3c2cc1[R]</smiles><smiles>[R]c1ccc2c([nH]c3ccccc32)c1[R]</smiles>

15

\section{Scheme 6}

同年，Jia 等 ${ }^{[11]}$ 研究一系列钉配合物[如 $\mathrm{RuCl}_{2}$ $\left(\mathrm{PPh}_{3}\right)_{3}, \mathrm{RuCl}_{2}(\mathrm{DMSO})_{4}, \mathrm{RuCl}_{3}, \mathrm{RuO}_{2},\left(\mathrm{NH}_{4}\right)_{2} \mathrm{RuCl}_{6}$ ]对有 机叠氮化合物的 $\mathrm{C}-\mathrm{H}$ 胺化反应的催化活性. 研究发现 $\mathrm{RuCl}_{3}$ 的催化活性最高，当叠氮基相对的芳环上有芳基 取代时有利反应进行，反应时间仅 $1.5 \mathrm{~h}$, 收率最高达 
表 2 环合的区域选择性

Table 2 Regioselectivity in forming the rings of carbazole

\begin{tabular}{cccc}
\hline R & $\mathrm{R}^{\prime}$ & $\begin{array}{c}\text { Regioselectivity } \\
(\mathbf{1 4}: \mathbf{1 5})\end{array}$ & Yield/\% \\
\hline $\mathrm{H}$ & $\mathrm{OMe}$ & $52: 84$ & 33 \\
$\mathrm{H}$ & $\mathrm{Me}$ & $66: 34$ & 25 \\
$\mathrm{H}$ & $\mathrm{F}$ & $89: 11$ & 65 \\
$\mathrm{H}$ & $\mathrm{Cl}$ & $>95: 5$ & 82 \\
$\mathrm{H}$ & $\mathrm{CF}_{3}$ & $>95: 5$ & 84 \\
$\mathrm{Me}$ & $\mathrm{F}$ & $39: 61$ & 59 \\
\hline
\end{tabular}

96\% (Scheme 7). 据作者推测在反应过程中, 有机叠氮 化合物先与钓催化剂进行反应形成复合物, 放出氮气后 形成中间体 $\mathbf{B}$, 可能经过两种重排途径转化成中间体 $\mathbf{D}$, 如 Scheme 7 所示, 而 $\mathbf{D}$ 是以咔唑为配体的钉配合物, 分 解后最终得到目标产物咔唑.
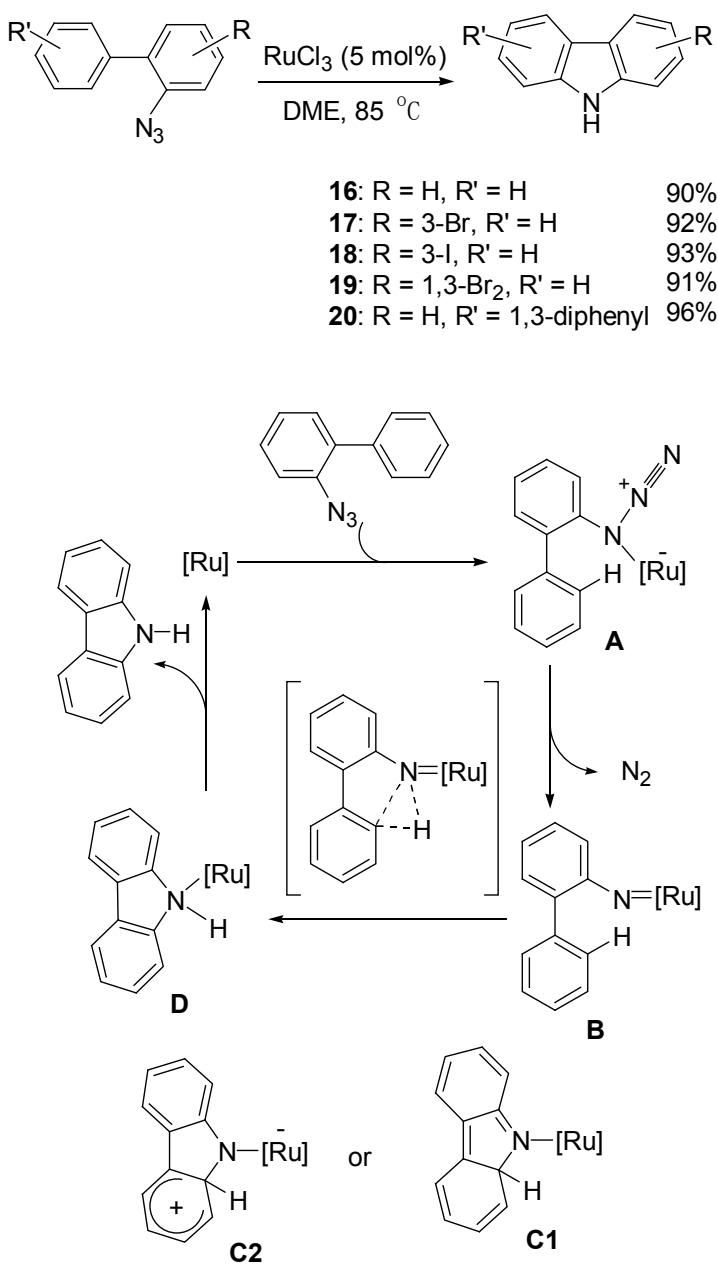

Scheme 7

近来，有报道指出胺基联苯同样也可用于咔唑的制 备, 特别是用于合成 $N$-取代不对称咔唑. 2007 年, Jean 等 ${ }^{[12]}$ 报道了以 $N$-取代胺基苯嘲酸醚和邻二卤代苯为原 料, 通过一锅法, 经 Suzuki-Miyaura 偶联反应和分子内 $\mathrm{S}_{\mathrm{N}} \mathrm{Ar}$ 反应直接合成咔唑(Scheme 8). $N$-取代基包括 Boc,
$\mathrm{Ms}, \mathrm{Ts}, \mathrm{Ac}, \mathrm{COCF}_{3}$, 实验结果显示只有取代基为磺酰基 (Ms 和 Ts)时产品全部为咔唑, 其他取代基仅得到相应 的联苯, 其中 $\mathrm{Ac}$ 和 $\mathrm{COCF}_{3}$ 取代时得到的联苯产品中约 有 $30 \% \sim 40 \%$ 产品的 $\mathrm{Ac}$ 和 $\mathrm{COCF}_{3}$ 被脱掉，提示此方法 实际用途有限.

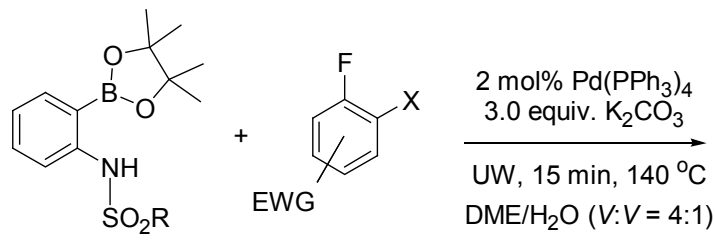

$(47 \% \sim 96 \%)$<smiles>[Y6]c1ccc2c3ccccc3n(S([R6])(=O)=O)c2c1</smiles><smiles>COC(=O)c1cc2c(cc1OC)[nH]c1ccccc12</smiles><smiles>[R]Nc1ccccc1B1OC(C)(C)C(C)(C)O1</smiles><smiles>[R]Nc1ccccc1-c1cc(C(C)=O)ccc1F</smiles>

Scheme 8

2008 年, Gaunt ${ }^{[13]}$ 和 Buchwald 小组 ${ }^{[14]}$ 分别使用 $\mathrm{Pd}(\mathrm{OAc})_{2}$ 为催化剂, 通过 $\mathrm{C}-\mathrm{H}$ 的胺化反应形成 $\mathrm{C}-\mathrm{N}$ 键，高收率地合成了一系列的不对称咔唑衍生物(Eq. 3, 表 3). 所合成的咔唑衍生物中不仅有取代基集中在其中 一个苯环上的，也有取代基分别分布在咔唑的两个苯环 上的，提示该方法可广泛用于合成不同多取代咔唑衍生 物.

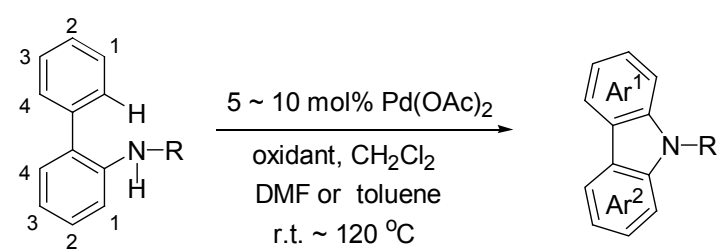


表 3 Eq. 3 中涉及的化合物和氧化剂

Table 3 Compounds and oxidants in Eq. 3

\begin{tabular}{|c|c|c|c|c|}
\hline $\mathrm{Ar}^{1}$ & $\mathrm{Ar}^{2}$ & $\mathrm{R}$ & Oxidant & Yield $/ \%$ \\
\hline $\mathrm{C}_{6} \mathrm{H}_{4}$ & $\mathrm{C}_{6} \mathrm{H}_{4}$ & Ac & $\mathrm{Cu}(\mathrm{OAc})_{2}$ & 92 \\
\hline $\mathrm{C}_{6} \mathrm{H}_{4}$ & $\mathrm{C}_{6} \mathrm{H}_{4}$ & $\mathrm{SO}_{2} \mathrm{Ph}$ & $\mathrm{Cu}(\mathrm{OAc})_{2}$ & 99 \\
\hline $\mathrm{C}_{6} \mathrm{H}_{4}$ & $\mathrm{C}_{6} \mathrm{H}_{4}$ & Boc & $\mathrm{Cu}(\mathrm{OAc})_{2}$ & 34 \\
\hline $\mathrm{C}_{6} \mathrm{H}_{4}$ & $\mathrm{C}_{6} \mathrm{H}_{4}$ & $\mathrm{COPh}$ & $\mathrm{Cu}(\mathrm{OAc})_{2}$ & $<10$ \\
\hline $\mathrm{C}_{6} \mathrm{H}_{4}$ & $\mathrm{C}_{6} \mathrm{H}_{4}$ & $\mathrm{COCF}_{2} \mathrm{CF}_{3}$ & $\mathrm{Cu}(\mathrm{OAc})_{2}$ & 0 \\
\hline $\mathrm{C}_{6} \mathrm{H}_{4}$ & $\mathrm{C}_{6} \mathrm{H}_{4}$ & $i-\operatorname{Pr}$ & $\mathrm{PhI}(\mathrm{OAc})_{2}$ & 96 \\
\hline $\mathrm{C}_{6} \mathrm{H}_{4}$ & $\mathrm{C}_{6} \mathrm{H}_{4}$ & $\mathrm{Me}$ & $\mathrm{PhI}(\mathrm{OAc})_{2}$ & 80 \\
\hline $\mathrm{C}_{6} \mathrm{H}_{4}$ & $\mathrm{C}_{6} \mathrm{H}_{4}$ & $t-\mathrm{Bu}$ & $\mathrm{PhI}(\mathrm{OAc})_{2}$ & 80 \\
\hline $\mathrm{C}_{6} \mathrm{H}_{4}$ & $\mathrm{C}_{6} \mathrm{H}_{4}$ & $\mathrm{Bn}$ & $\mathrm{PhI}(\mathrm{OAc})_{2}$ & 96 \\
\hline $\mathrm{C}_{6} \mathrm{H}_{4}$ & $1,3-\mathrm{Me}_{2}-\mathrm{C}_{6} \mathrm{H}_{2}$ & Ac & $\mathrm{Cu}(\mathrm{OAc})_{2}$ & 90 \\
\hline $\mathrm{C}_{6} \mathrm{H}_{4}$ & $1,3-\mathrm{F}_{2}-\mathrm{C}_{6} \mathrm{H}_{2}$ & Ac & $\mathrm{Cu}(\mathrm{OAc})_{2}$ & 94 \\
\hline $\mathrm{C}_{6} \mathrm{H}_{4}$ & $3-\mathrm{Me}-\mathrm{C}_{6} \mathrm{H}_{3}$ & Ac & $\mathrm{Cu}(\mathrm{OAc})_{2}$ & 92 \\
\hline $\mathrm{C}_{6} \mathrm{H}_{4}$ & $3-\mathrm{F}-\mathrm{C}_{6} \mathrm{H}_{3}$ & Ac & $\mathrm{Cu}(\mathrm{OAc})_{2}$ & 94 \\
\hline $\mathrm{C}_{6} \mathrm{H}_{4}$ & $3-\mathrm{CF}_{3}-\mathrm{C}_{6} \mathrm{H}_{3}$ & Ac & $\mathrm{Cu}(\mathrm{OAc})_{2}$ & 88 \\
\hline 4-MeO- $\mathrm{C}_{6} \mathrm{H}_{3}$ & $\mathrm{C}_{6} \mathrm{H}_{4}$ & Ac & $\mathrm{Cu}(\mathrm{OAc})_{2}$ & 81 \\
\hline $4-\mathrm{F}-\mathrm{C}_{6} \mathrm{H}_{3}$ & $\mathrm{C}_{6} \mathrm{H}_{4}$ & Ac & $\mathrm{Cu}(\mathrm{OAc})_{2}$ & 78 \\
\hline $4-\mathrm{CF}_{3}-\mathrm{C}_{6} \mathrm{H}_{3}$ & $\mathrm{C}_{6} \mathrm{H}_{4}$ & Ac & $\mathrm{Cu}(\mathrm{OAc})_{2}$ & 81 \\
\hline $2,4-(\mathrm{MeO})_{2}-\mathrm{C}_{6} \mathrm{H}_{2}$ & $\mathrm{C}_{6} \mathrm{H}_{4}$ & $\mathrm{Ac}$ & $\mathrm{Cu}(\mathrm{OAc})_{2}$ & 85 \\
\hline $2-\mathrm{MeO}-\mathrm{C}_{6} \mathrm{H}_{3}$ & $3-\mathrm{Me}-\mathrm{C}_{6} \mathrm{H}_{3}$ & $\mathrm{Ac}$ & $\mathrm{Cu}(\mathrm{OAc})_{2}$ & 88 \\
\hline $3-\mathrm{MeO}-\mathrm{C}_{6} \mathrm{H}_{3}$ & $\mathrm{C}_{6} \mathrm{H}_{4}$ & $\mathrm{Bn}$ & $\mathrm{PhI}(\mathrm{OAc})_{2}$ & 85 \\
\hline $3-\mathrm{Me}-\mathrm{C}_{6} \mathrm{H}_{3}$ & $\mathrm{C}_{6} \mathrm{H}_{4}$ & $\mathrm{Bn}$ & $\mathrm{PhI}(\mathrm{OAc})_{2}$ & 86 \\
\hline $3-\mathrm{F}-\mathrm{C}_{6} \mathrm{H}_{3}$ & $\mathrm{C}_{6} \mathrm{H}_{4}$ & $\mathrm{Bn}$ & $\mathrm{PhI}(\mathrm{OAc})_{2}$ & 72 \\
\hline $3-\mathrm{Cl}-\mathrm{C}_{6} \mathrm{H}_{3}$ & $\mathrm{C}_{6} \mathrm{H}_{4}$ & $\mathrm{Bn}$ & $\mathrm{PhI}(\mathrm{OAc})_{2}$ & 80 \\
\hline $3-\mathrm{MeO}_{2} \mathrm{C}-\mathrm{C}_{6} \mathrm{H}_{3}$ & $\mathrm{C}_{6} \mathrm{H}_{4}$ & $\mathrm{Bn}$ & $\mathrm{PhI}(\mathrm{OAc})_{2}$ & 95 \\
\hline $3-\mathrm{NO}_{2}-\mathrm{C}_{6} \mathrm{H}_{3}$ & $\mathrm{C}_{6} \mathrm{H}_{4}$ & $\mathrm{Bn}$ & $\mathrm{PhI}(\mathrm{OAc})_{2}$ & 72 \\
\hline $2-\mathrm{MeO}-\mathrm{C}_{6} \mathrm{H}_{3}$ & $\mathrm{C}_{6} \mathrm{H}_{4}$ & $\mathrm{Bn}$ & $\mathrm{PhI}(\mathrm{OAc})_{2}$ & 81 \\
\hline $2-\mathrm{MeO}_{2} \mathrm{C}-\mathrm{C}_{6} \mathrm{H}_{3}$ & $\mathrm{C}_{6} \mathrm{H}_{4}$ & $\mathrm{Bn}$ & $\mathrm{PhI}(\mathrm{OAc})_{2}$ & 94 \\
\hline $4-\mathrm{MeO}-\mathrm{C}_{6} \mathrm{H}_{3}$ & $\mathrm{C}_{6} \mathrm{H}_{4}$ & $\mathrm{Bn}$ & $\mathrm{PhI}(\mathrm{OAc})_{2}$ & 75 \\
\hline 4-Me- $\mathrm{C}_{6} \mathrm{H}_{3}$ & $\mathrm{C}_{6} \mathrm{H}_{4}$ & $\mathrm{Bn}$ & $\mathrm{PhI}(\mathrm{OAc})_{2}$ & 56 \\
\hline $\mathrm{C}_{6} \mathrm{H}_{4}$ & $1-\mathrm{Me}-\mathrm{C}_{6} \mathrm{H}_{3}$ & $\mathrm{Bn}$ & $\mathrm{PhI}(\mathrm{OAc})_{2}$ & 81 \\
\hline $\mathrm{C}_{6} \mathrm{H}_{4}$ & $2-\mathrm{MeO}-\mathrm{C}_{6} \mathrm{H}_{3}$ & $\mathrm{Bn}$ & $\mathrm{PhI}(\mathrm{OAc})_{2}$ & 89 \\
\hline $\mathrm{C}_{6} \mathrm{H}_{4}$ & $2-\mathrm{CF}_{3}-\mathrm{C}_{6} \mathrm{H}_{3}$ & $\mathrm{Bn}$ & $\mathrm{PhI}(\mathrm{OAc})_{2}$ & 85 \\
\hline $\mathrm{C}_{6} \mathrm{H}_{4}$ & $3-\mathrm{Cl}-\mathrm{C}_{6} \mathrm{H}_{3}$ & $\mathrm{Bn}$ & $\mathrm{PhI}(\mathrm{OAc})_{2}$ & 64 \\
\hline $\mathrm{C}_{6} \mathrm{H}_{4}$ & $3-\mathrm{Me}-\mathrm{C}_{6} \mathrm{H}_{3}$ & $\mathrm{Bn}$ & $\mathrm{PhI}(\mathrm{OAc})_{2}$ & 89 \\
\hline $\mathrm{C}_{6} \mathrm{H}_{4}$ & $3-\mathrm{F}-\mathrm{C}_{6} \mathrm{H}_{3}$ & $\mathrm{Bn}$ & $\mathrm{PhI}(\mathrm{OAc})_{2}$ & 70 \\
\hline $\mathrm{C}_{6} \mathrm{H}_{4}$ & $3-\mathrm{MeO}_{2} \mathrm{C}-\mathrm{C}_{6} \mathrm{H}_{3}$ & $\mathrm{Bn}$ & $\mathrm{PhI}(\mathrm{OAc})_{2}$ & 60 \\
\hline $\mathrm{C}_{6} \mathrm{H}_{4}$ & 4-Me- $\mathrm{C}_{6} \mathrm{H}_{3}$ & $\mathrm{Bn}$ & $\mathrm{PhI}(\mathrm{OAc})_{2}$ & 83 \\
\hline
\end{tabular}

同年, Shi 等 ${ }^{[15]}$ 开发了一种新的偶联方式, 以 2,3-二 甲基-4-甲氧基乙酰苯胺和苯为原料, 以 $\mathrm{Pd}(\mathrm{OAc})_{2}$ 和 $\mathrm{Cu}(\mathrm{OTf})_{2}$ 为催化剂活化 $\mathrm{C}-\mathrm{H}$ 键, 从而直接偶联得到联 苯, 再以 $\mathrm{Pd}(\mathrm{OAc})_{2}$ 和 $\mathrm{Cu}(\mathrm{OAc})_{2}$ 为催化剂合成咔唑 (Scheme 9), 虽然第一步收率并不高, 但这是一种全新 的偶联方式, 与 Suzuki-Miyaura 偶联反应相比, 其优势 在于免去了在原料特定位置引入硣酸和卤素取代基的 麻烦，但该方法在环合时的区域选择性问题还有待研 究.

Nishiyama 等 ${ }^{[16]}$ 系统研究了高价碘化物 $\mathrm{PhI}\left(\mathrm{OCH}_{2}-\right.$ $\left.\mathrm{CF}_{3}\right)_{2}$ 诱导的氧化环合反应在咔唑合成中的应用. 研究 发现, 与产品化的双(三氟乙酰氧基)碘苯[Phenyliodine
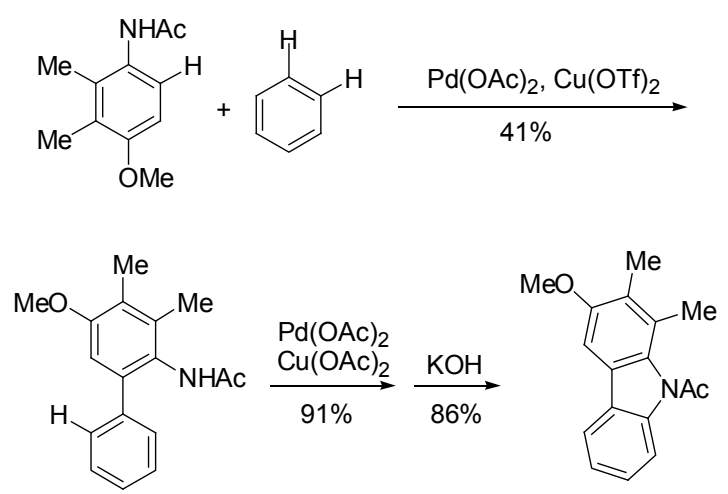

Scheme 9

(III) bis(trifluoroacetate), PIFA]相比, 新型高价碘化物 $\mathrm{PhI}\left(\mathrm{OCH}_{2} \mathrm{CF}_{3}\right)_{2}$ 在较温和的条件下即可高效地得到咔唑 
产物(Eq. 4, 表 4), 而 PIFA 在低温反应时收率较低, 且 在联苯中间体上带有不同取代基时会有副产物生成.<smiles>[R]c1cc(N)c(-c2ccc([R])c([R])c2[R])cc1[R]</smiles>

$$
\underset{\mathrm{TFE}}{\stackrel{\mathrm{Phl}\left(\mathrm{OCH}_{2} \mathrm{CF}_{3}\right)_{2}}{\longrightarrow}}
$$

21

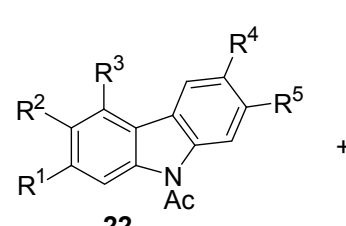<smiles>[R]c1ccc2c(c1)[nH]c1cc([R])c([R])cc12</smiles>

22

$\mathrm{R}^{1} \sim \mathrm{R}^{5}=\mathrm{H}$

$\mathrm{R}^{1}=\mathrm{OMe}, \mathrm{R}^{2} \sim \mathrm{R}^{5}=\mathrm{H} \quad 73 \%$

$R^{2}=$ OMe, $R^{1}, R^{3} \sim R^{5}=H \quad 91 \%$

$R^{3}=O M e, R^{1} \sim R^{2}, R^{4} \sim R^{5}=H^{40 \%}$

$\mathrm{R}^{4}=\mathrm{OMe}, \mathrm{R}^{1} \sim \mathrm{R}^{3}, \mathrm{R}^{5}=\mathrm{H} \quad 0 \%$

$\mathrm{R}^{5}=\mathrm{OMe}, \mathrm{R}^{1} \sim \mathrm{R}^{4}=\mathrm{H} \quad 83 \%$

$R^{2}=R^{5}=O M e, R^{1}, R^{3}, R^{4}=H \quad 44 \%$

表 4 不同反应条件和收率

Table 4 Different reagents, conditions and yields<smiles>COc1cccc(-c2cc(C)ccc2NC(=O)C(F)(F)F)c1</smiles>

\begin{tabular}{cllc}
\hline Entry & Reagent & Condition & Yield/\% \\
\hline 1 & PhI $\left(\mathrm{OCH}_{2} \mathrm{CF}_{3}\right)_{2}$ & r.t. & 87 \\
2 & PIFA & $0{ }^{\circ} \mathrm{C}$ & 58 \\
3 & C.P.E. & r.t. & 0 \\
\hline
\end{tabular}

${ }^{a}$ Recovered starting materials.

Chang 等 ${ }^{[17]}$ 最近报道了 $\mathrm{Cu}$ 催化分子内氧化偶联形 成 $\mathrm{C}-\mathrm{N}$ 的合成方法, 可以广泛用于咔唑合成. 其合成 的起始原料为苯磺酰胺取代的联苯, 在三氟醋酸存在条 件下经 $\mathrm{Cu}(\mathrm{OTf})_{2}$ 催化, 反应 $10 \mathrm{~min}$ 即可以大于 $90 \%$ 的 收率得到咔唑(Eq. 5). 此方法较之前述方法在操作及收 率方面无疑更具优势，但该方法是否可推广用于合成 单/多取代的咔唑衍生物，该报道未有探讨.

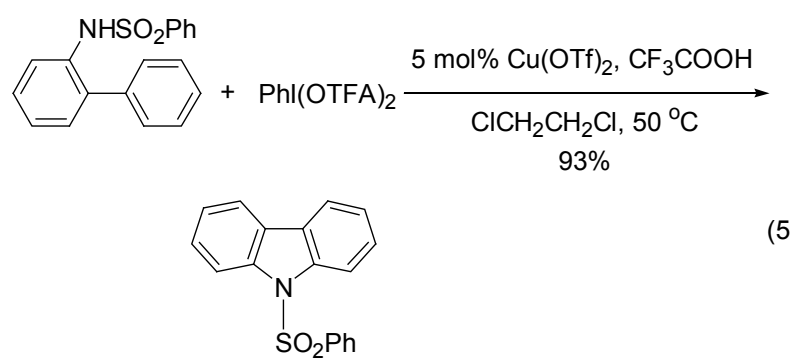

以双卤素或羟基取代联苯为原料同样可以合成卡 唑. 2008 年, Chida 小组 ${ }^{[18]}$ 应用双卤素取代的联苯和伯胺 为原料，通过分子内 Friedel-Crafts 型迈克尔加成反应和 $\mathrm{Pd}$ 催化 $\mathrm{C}-\mathrm{O}$ 偶联全合成了 (土)-Murrayazoline, 其关键 步骤之一即为咔唑环的构建(Scheme 10).
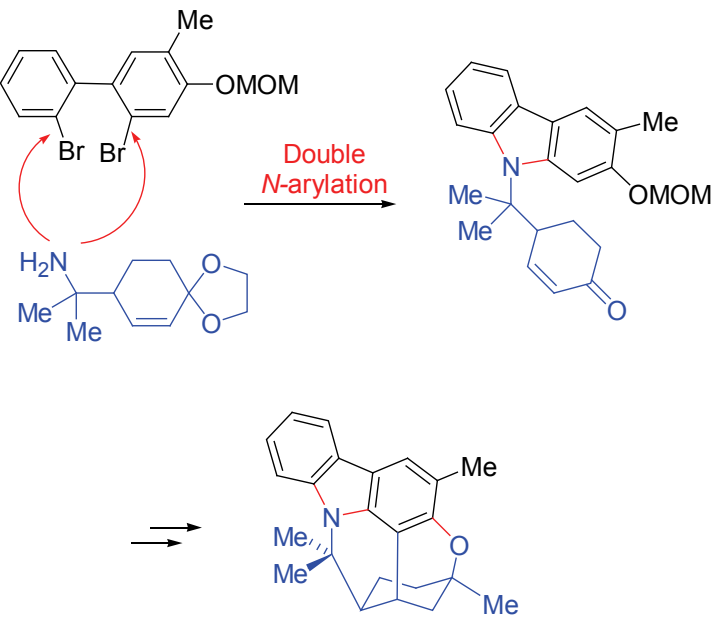

Scheme 10

2009 年, $\mathrm{Li}$ 等 ${ }^{[19]}$ 用类似的方法合成了一系列对称 $N$-取代咔唑及其衍生物，收率 53\%～98\% (Eq. 6). 实验 结果显示取代基体积越大、数目越多收率就越低. 而 Nozaki 小组 ${ }^{[20]}$ 以双羟基联苯为原料, 先用 $\mathrm{Tf}_{2} \mathrm{O}$ 保护分 子中的羟基，再以 $\mathrm{Pd}$ 配合物为催化剂，通过分子内 Friedel-Crafts 型迈克尔加成反应合成 11-苯基苯并呋喃[3,2-b]-咔唑(Eq. 7)
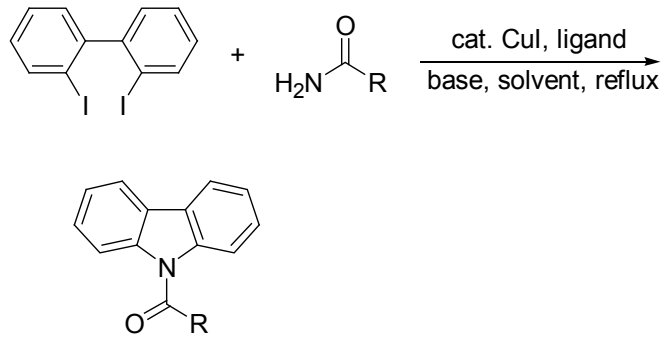

24: $\mathrm{R}=\mathrm{Bu}, 98 \%$

25: $\mathrm{R}=\mathrm{OEt}, 69 \%$

26: $\mathrm{R}=4-\mathrm{MeOC}_{6} \mathrm{H}_{4}, 89 \%$

27: $\mathrm{R}=4-\mathrm{H}_{2} \mathrm{NC}_{6} \mathrm{H}_{4}, 82 \%$

\section{2 以二苯胺为中间体的方法}

以二苯胺为中间体同样是合成咔唑的常用策略. 与 联苯方法相反，其先是构建 $\mathrm{C}-\mathrm{N}$ 键，然后经 Suzuki 等 反应构建 $\mathrm{C}-\mathrm{C}$ 键合成咔唑. $\mathrm{C}-\mathrm{C}$ 的构建可经直接偶联 获得, 即不需要特定的取代基, 直接依靠 $\mathrm{C}-\mathrm{H}$ 进行偶 联, 如 Eq. 8 所示. 

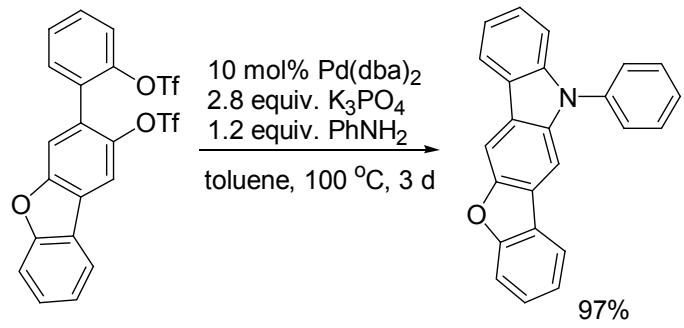

(7)

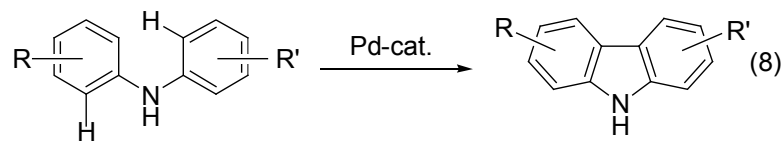

2007 年, Ohno 小组 ${ }^{[21]}$ 以 $\mathrm{Tf}_{2} \mathrm{O}$ 保护的取代苯酚和取 代苯胺为原料, 以 $\mathrm{CsCO}_{3}$ 和 $\mathrm{Pd}(\mathrm{OAc})_{2}$ 为催化剂, 使用一 锅法, 先通过 $N$-芳基化反应生成取代二苯胺中间体, 再 与催化剂 $\mathrm{Pd}(\mathrm{OAc})_{2}$ 作用, 形成有机金属化合物. $\mathrm{Pd}(\mathrm{II})$ 被还原成 $\operatorname{Pd}(0)$ 的同时, 偶联形成分子内 $\mathrm{C}-\mathrm{C}$, 最终合 成了 12 个不对称的咔唑衍生物. 结果显示, 该法合成的 咔坐及衍生物收率主要在 $60 \% \sim 80 \%$ 之间, 个别高达 90\% 99\% (Scheme 11). 随后, 他们又对催化体系进行 了系统研究与完善 ${ }^{[22]}$, 合成了大量的咔唑衍生物, 发现 二芳胺的取代基对氧化偶联有重要影响, 并从动力学同 位素效应实验中获得的反应机理方面的重要信息, 详细 阐述了其氧化偶联的反应机理. 2008 年, Fagnou 小组 ${ }^{[23]}$
改进了分子内 $\mathrm{Pd}$ 催化氧化 $\mathrm{C}-\mathrm{C}$ 键生成反应，用新戊酸 代替了醋酸, 以 $\mathrm{Pd}(\mathrm{OAc})_{2}$ 为催化剂合成了一系列咔唑 及衍生物, 经过改进反应具有更好的重现性, 更高的收 率(Eq. 9). 2009 年, Menéndez 小组 ${ }^{[24]}$ 对前人工作进行了 改进, 开发了非酸性条件微波辅助分子内 $\mathrm{Pd}$ 催化氧化 $\mathrm{C}-\mathrm{C}$ 键生成反应合成咔唑的方法(Eq. 10). 研究发现, 该方法并未因在非酸性条件下使产品的收率发生严重 下降, 并且拓展了分子内 Pd 催化氧化芳环偶联的范围.

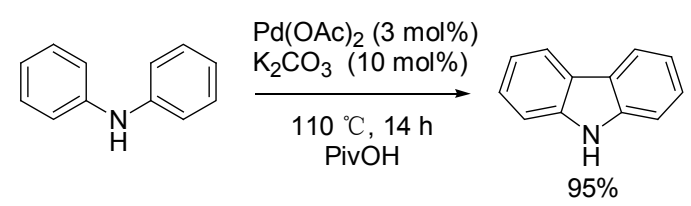

2008 年, Chatani 等 ${ }^{[25]}$ 利用 2-氧基和 $2^{\prime}$-氯取代的二 苯胺和二苯醚, 通过 $\mathrm{Rh}$ 催化硅烷化反应合成了咔唑和 二苯并呋喃等化合物，合成咔唑的收率为 55\% (Eq. 11). 虽然收率不高, 催化体系较为复杂, 但同样为合成咔唑 提供了一条路径. 2009 年, Bedford 小组 ${ }^{[26]}$ 以 2-卤代$2^{\prime}, 5^{\prime}$-烷基取代二苯胺为原料, $\mathrm{Pd}(\mathrm{OAc})_{2}$ 催化剂, 催化合 成了多个咔唑衍生物, 由于产物较为复杂, 因此收率较 低, 有时甚至无法得到目标产物(Eq. 12). Miura 等 ${ }^{[27]}$ 以 2-芳基苯甲酸为原料, 利用 $\mathrm{Rh} / \mathrm{Cu}$ 催化体系, 合成了 4-

$$
\text { toluene }
$$

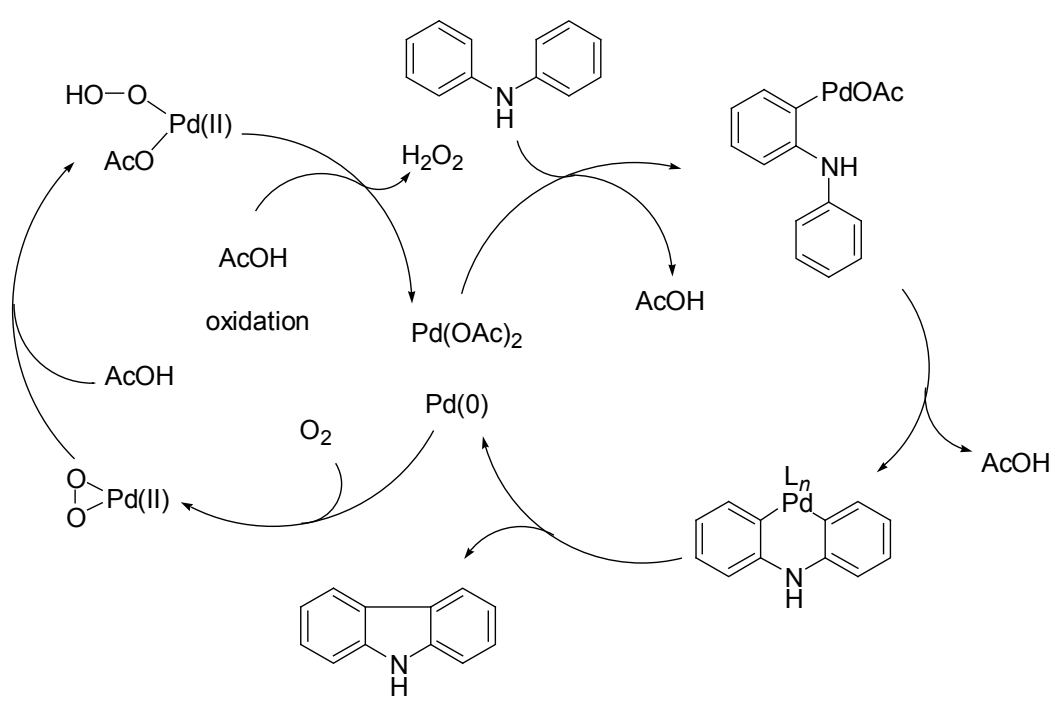

Scheme 11 
<smiles>[R]c1ccc(Nc2ccc([R3])c([R])c2[R])cc1</smiles><smiles>[R]c1ccc2[nH]c3c([R])c([R])c([R])cc3c2c1</smiles>

28: $R^{1}=R^{2}=R^{3}=R^{4}=H, 84 \%$

29: $R^{2}=O M e, R^{1}=R^{3}=R^{4}=H, 84 \%$

30: $R^{3}=$ OMe, $R^{1}=R^{2}=R^{4}=H, 86 \%$

31: $R^{1}=R^{4}=O M e, R^{2}=R^{3}=H, 89 \%$

32: $R^{2}=R^{4}=O M e, R^{1}=R^{3}=H, 90 \%$

33: $R^{3}=F, R^{4}=$ OMe, $R^{1}=R^{2}=H, 85 \%$

34: $R^{2}=$ OEt, $R^{4}=$ OMe, $R^{1}=R^{3}=H, 91 \%$

35: $R^{2}=M e, R^{4}=O M e, R^{1}=R^{3}=H, 83 \%$

36: $R^{2}=O M e, R^{4}=M e, R^{1}=R^{3}=H, 85 \%$

37: $R^{2}=$ OEt, $R^{4}=M e, R^{1}=R^{3}=H, 80 \%$

乙烯基咔唑和异香豆素衍生物, 通过选用不同的催化剂 可以对产物以及比例进行控制(Scheme 12).<smiles>N#Cc1ccccc1Nc1ccccc1Cl</smiles>

cat. [RhCl(cod) $]_{2}$ $\mathrm{Me}_{3} \mathrm{Si}-\mathrm{SiMe}_{3}, 2$ equiv. $130{ }^{\circ} \mathrm{C}, 15 \mathrm{~h}$

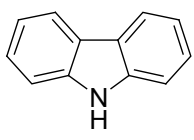

$55 \%$<smiles>[R][R]1ccc2[nH]c3c(c2c1)C=C[R1](CC(C)C)C=C3</smiles>

$0 \% \sim 62 \%$

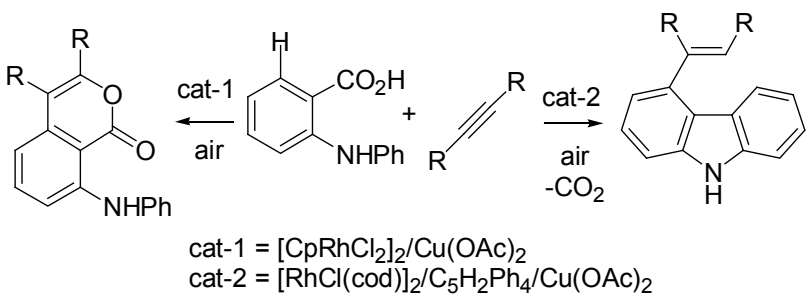

Scheme 12

2009 年, Rossi 等 ${ }^{[28]}$ 以 2-氯代或 2-溴代的二苯胺为 原料, 在叔丁醇钾存在下, 通过光催化 $\mathrm{S}_{\mathrm{RN}} 1$ 意思取代反 应合成了一系列的咔唑及衍生物. 研究发现该方法的收 率均高达 $90 \%$ 以上, 仅需 $350 \mathrm{~nm}$ 紫外光源, 相比需要贵 金属催化的方法优势明显，值得我们重点关注(Eq. 13).
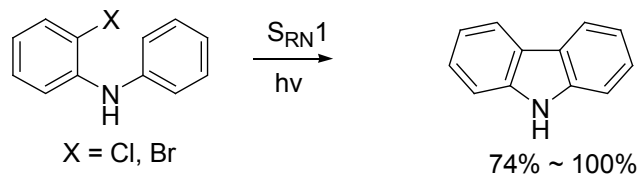

2010 年, Buchwald 等 ${ }^{[29]}$ 以 2-乙烯基-2'-甾代二苯胺 为关键中间体, 经钯催化剂催化反应, 成功合成了 1 -乙

烯基取代咔唑衍生物，产率达 78\%～94\% (Eq. 14). 研 究还发现，通过选用不同的钯催化剂配体，还可合成二 苯并氮杂草以及 9-甲基呋啶等产物.<smiles>[R]c1cccc2c1[nH]c1c(C=C)cccc12</smiles>

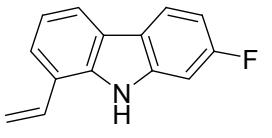

$78 \%$<smiles>C=Cc1cccc2c1[nH]c1cc(C)ccc12</smiles><smiles>C=Cc1cccc2c1[nH]c1ccccc12</smiles>

$94 \%$<smiles>C=Cc1cccc2c1[nH]c1cc(C(F)(F)F)ccc12</smiles><smiles>C=Cc1cccc2c1[nH]c1ccc(C)cc12</smiles>

\section{3 以吲哚为中间体的方法}

以吲哚为中间体通过引入第二芳基同样可以合成 咔坐.

Diels-Alder 反应在咔唑构建过程中有着广泛的应 用. 2007 年, Rossi 小组 ${ }^{[30]}$ 先以 3-乙烯基吲哚为原料, 通 过 Diels-Alder 反应合成了几个手性咔唑衍生物，收率不 高，通常在 $40 \% \sim 80 \%$ 之间(Eq. 15). 随后，Bernardi 小 组 ${ }^{[31]}$ 于 2008 年同样以 3-乙烯基吲哚为原料, 催化合成 了一系列手性咔唑衍生物，收率提高至 70\% 90\%之间， 同时 $e e$ 值均在 $90 \%$ 以上，部分化合物甚至高达 $99 \%$ (Eq. 16). 二者同样是通过 Diels-Alder 反应合成咔唑衍生物, 在溶剂、温度和反应时间等条件方面虽有差别，但收率 和 $e e$ 值的提高主要归因于催化剂的使用.

2009 年, Tao 等 ${ }^{[32]}$ 以 5-(2-1H-吲哚)-2-甲基-6,7-2H吲唑和顺-3-氧基丙烯酸乙酯为原料, $\mathrm{YtBr}_{3}$ 为催化剂, 通过区域选择性 Diels-Alder 反应和催化脱氢合成了几 个咔唑衍生物, 路线如 Scheme 13 所示.

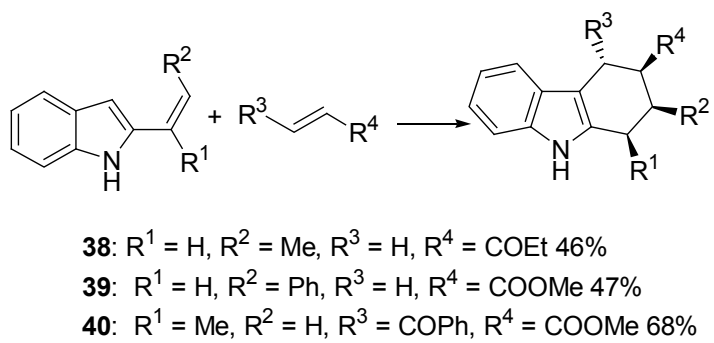



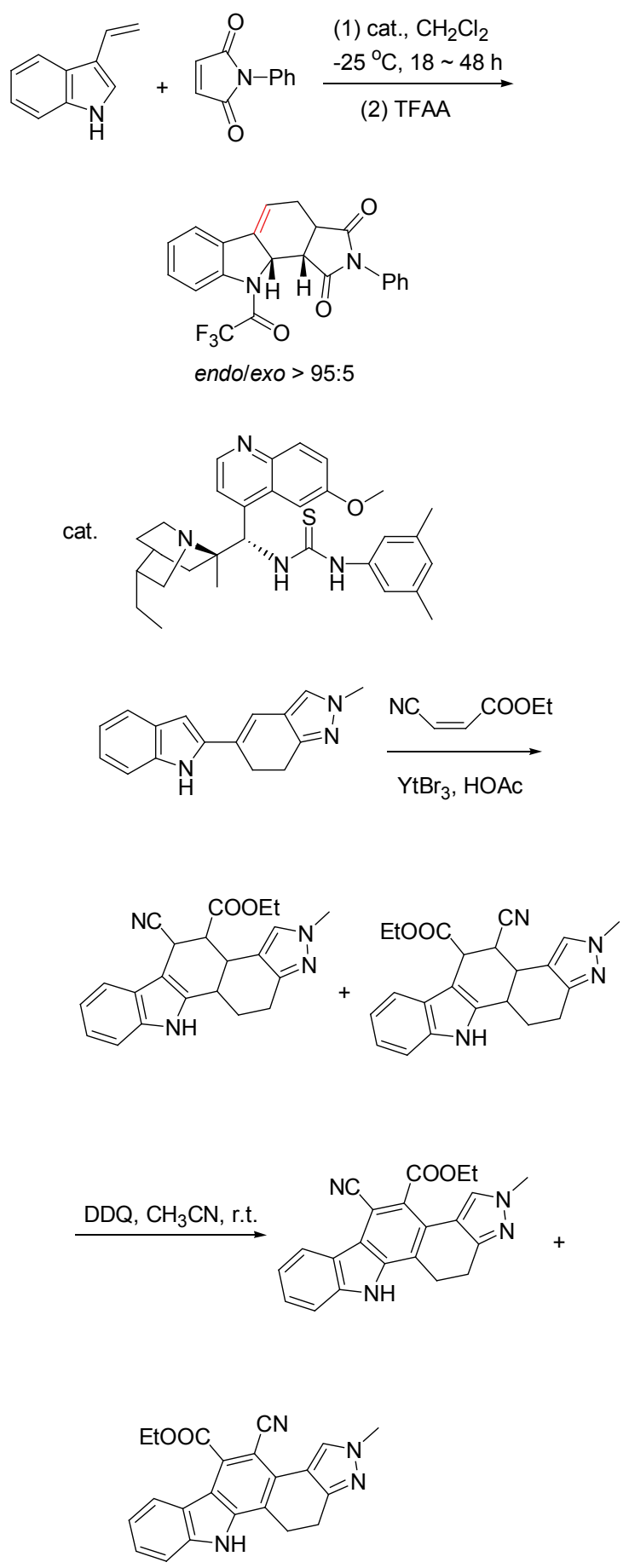

\section{Scheme 13}

2008 年, Sáa 等 ${ }^{[33]}$ 报道了一种新的 Diels-Alder 反应: 分子内脱氢 Diels-Alder 反应, 并成功将其用于咔唑衍生 物的合成, 合成策略如 Scheme 14 所示. 2009 年, Witulski 等 ${ }^{[34]}$ 在全合成 antiostatin $A_{1}$ 的过程中, 就利用分 子内脱氢 Diels-Alder 反应, 使用 $\mathrm{RhCl}\left(\mathrm{PPh}_{3}\right)_{3}$ 催化剂合 成了 $N$-Ts-1-正戊基-2-甲基-3-甲氧基咔唑中间体, 收率 达到 $82 \%$ (Eq. 17).
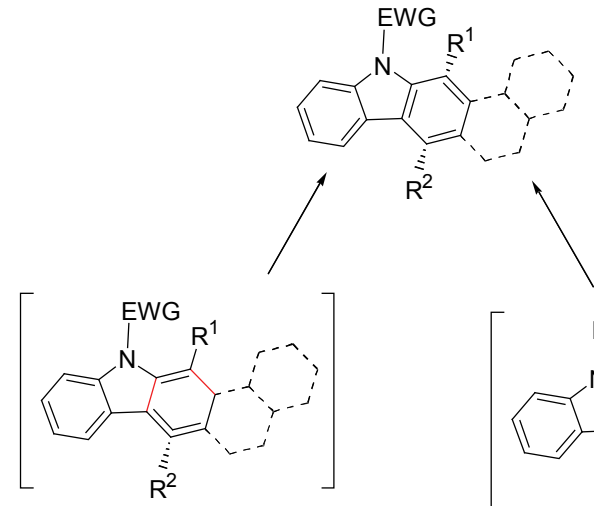

$\uparrow \triangle$

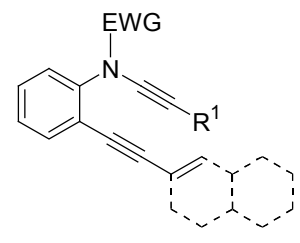

$\mathrm{EWG}=\mathrm{Ts}, \mathrm{Ms}, \mathrm{CO}_{2} \mathrm{Me}$ $\mathrm{R}^{1}=\mathrm{H}, \mathrm{TMS}$

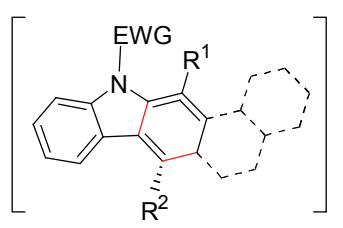
$\uparrow \triangle$<smiles>[R]C#Cc1ccccc1N(C#CC1=CCCN2CCCCC12)C(C)(C)C</smiles>

$\mathrm{R}^{2}=\mathrm{H}, \mathrm{TMS}, \mathrm{Ph}$

\section{Scheme 14}

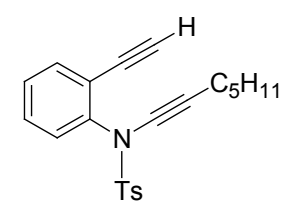

$\mathrm{Ch}_{3} \mathrm{O}=\mathrm{CH}_{3}$

$\mathrm{RhCl}\left(\mathrm{PPh}_{3}\right)_{3}(10 \mathrm{~mol} \%)$

toluene, r.t., $2 \mathrm{~d}$

$82 \%$

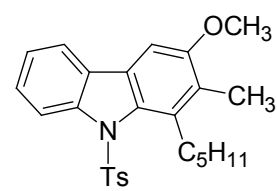

2008 年, Shirakawa 等 ${ }^{[35]}$ 选择与上述 Diels-Alder 反 应相似的原料, 以 $\mathrm{In}(\mathrm{ONf})_{3}$ 为催化剂, 通过催化偶联反 应，合成了 6-甲基- $11 H$-苯并 $[a]$ 咔唑一系列的咔唑衍生 物(Eq. 18). 同年, Routier 小组 ${ }^{[36]}$ 用 $N$-甲基-3,4-二溴丁二 酰亚胺代替炔烃，在 LiHMDS/THF 条件下，合成了中间 体 41, 然后使用 $\mathrm{Pd}(\mathrm{OAc})_{2}$ 催化反应得到 4 个咔唑衍生 物, 合成路线如 Scheme 15 所示.

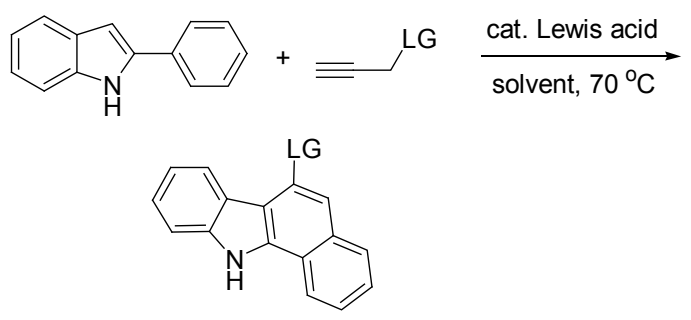


<smiles>[R]c1cc([R])c([R])c(-c2cc3ccccc3[nH]2)c1</smiles><smiles>CN1C(=O)C(Br)=C(Br)C1=O</smiles><smiles>[R]c1cc([R])c([R])c(-c2[nH]c3ccccc3c2C2=C(Br)C(=O)N(C)C2=O)c1</smiles>

41

$$
\begin{aligned}
& R^{1}=H, R^{2}=R^{3}=H \quad 75 \% \\
& R^{1}=F, R^{2}=R^{3}=H \quad 92 \% \\
& R^{1}=C N, R^{2}=R^{3}=H \quad 75 \% \\
& R^{1}=N_{2}, R^{2}=R^{3}=H \quad 65 \% \\
& R^{1}=H, R^{2}=R^{3}=M e \quad 38 \%
\end{aligned}
$$

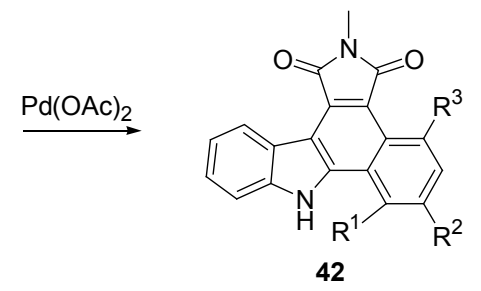

$$
\begin{aligned}
& R^{1}=H, R^{2}=R^{3}=H \quad 70 \% \\
& R^{1}=F, R^{2}=R^{3}=H \quad 78 \% \\
& R^{1}=C N, R^{2}=R^{3}=H \quad-- \\
& R^{1}=N_{2}, R^{2}=R^{3}=H \quad 36 \% \\
& R^{1}=H, R^{2}=R^{3}=\operatorname{Me} \quad 80 \%
\end{aligned}
$$

\section{Scheme 15}

2009 年, Miura 小组 ${ }^{[37,38]}$ 利用 Pd 催化氧化偶联反应, 以 $N$-甲基吲哚-3-甲酸与两分子炔烃反应合成了 1,2,3,4四取代咔唑(Eq. 19). 同年, 他们采用同样方法用 $N$-甲 基吲哚代替了吲哚-3-甲酸为原料合成了 1,2,3,4-四取代 咔唑, 但是反应的收率大幅降低. 随着研究的深入, 他 们用 $N$-甲基吡咯-2-甲酸代替 $N$-甲基吲哚合成了 1,2,3,4,5,6,7,8-八取代咔唑(Scheme 16). 2008 年, Zhang 等 ${ }^{[39]}$ 以 $N$-取代-2-氯-3-甲酰基吲哚和取代苯乙烯为原料, 通过光催化一锅法合成了一系列苯并咔唑, 收率波动范 围在 $25 \% \sim 82 \%$ (Eq. 20). 根据作者对反应机理的阐述, 该方法先进行光催化脱氯偶联, 然后通过电环化完成关 环.

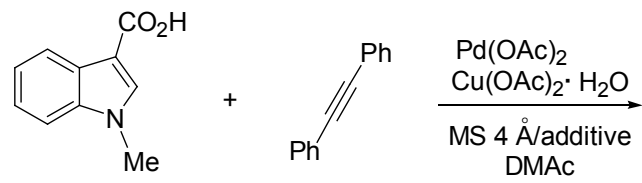<smiles>Cn1c2ccccc2c2c(-c3ccccc3)c(-c3ccccc3)c(-c3ccccc3)c(-c3ccccc3)c21</smiles><smiles>[R]C#CCC#CC[R]</smiles><smiles>[R]c1c([R])c([R])c2c(c1[R])c([R])n1c([R])c([R])c([R])c([R])c21</smiles>

$64 \% \sim 82 \%$

Scheme 16<smiles>[R]C=C([R])c1ccc([R7])cc1</smiles><smiles></smiles>

$$
\begin{aligned}
& \text { 43: } R^{1}=P h, R^{2}=R^{3}=R^{4}=H \quad 70 \% \\
& \text { 44: } R^{1}=P h, R^{3}=M e, R^{2}=R^{4}=H \quad 41 \% \\
& \text { 45: } R^{1}=P h, R^{3}=P h, R^{2}=R^{4}=H \quad 82 \% \\
& \text { 46: } R^{1}=P h, R^{2}=M e, R^{3}=H, R^{4}=O M e \quad 36 \% \\
& \text { 47: } R^{1}=M e, R^{2}=R^{3}=R^{4}=H \quad 54 \% \\
& \text { 48: } R^{1}=M e, R^{3}=M e, R^{2}=R^{4}=H \quad 35 \% \\
& \text { 49: } R^{1}=M e, R^{3}=P h, R^{2}=R^{4}=H \quad 65 \% \\
& \text { 50: } R^{1}=M e, R^{2}=M e, R^{3}=H, R^{4}=O M e \quad 45 \%
\end{aligned}
$$

2008 年, Tiano 和 Belmont 等 ${ }^{[40]}$ 选择带有甲基酮和 分子内炔烃的吲哚作为前体, 以 $4 \AA$ 分子笁为催化剂, 通过 Aminobenzannulation 反应合成了胺基取代的 6 个 咔唑衍生物，反应收率在 65\% 96\%之间(Eq. 21). 2009 年, Rossi 等 ${ }^{[41]}$ 开发新的 Lewis 酸催化体系，选择与 Belmont 相似的原料, 并使用该催化体系合成了 6 个结 构更为复杂的咔唑衍生物(Eq. 22).

2007 年, Ila 等 ${ }^{[42]}$ 以环丙烷取代吲哚为原料, 通过 Domino 碳正离子重排, Lewis 酸催化成功合成了几个咔 唑衍生物，该反应产物复杂，受反应体系影响很大，当 采用 PTSA $/ \mathrm{C}_{6} \mathrm{H}_{6}$ 时得到的两个咔唑衍生物的分别达到 了 $51 \%$ 和 65\% (Scheme 17)，尽管如此，反应收率和产物 控制仍有待研究. 
<smiles>CC(=O)c1c(C#Cc2ccc(C)cc2)n(C)c2ccccc12</smiles><smiles>Cn1c2ccccc2c2c(N3CCCC3)cc(-c3ccccc3)cc21</smiles><smiles>[R]C#CC([GeH2])c1c(C(C)=O)[nH]c2ccccc12</smiles>

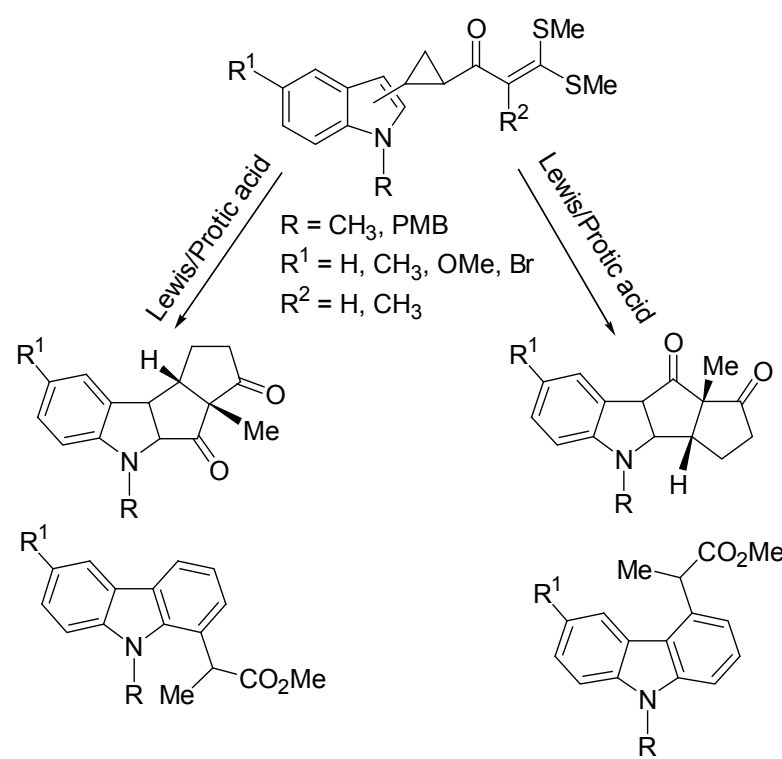

Scheme 17

2007 年, Padwa 等 ${ }^{[43]}$ 利用 $\mathrm{Rh}$ 催化 Domino 反应作为 关键步骤, 合成了 kopsifoline 生物碱框架, 该化合物是 一个相当复杂的咔唑衍生物, 其关键步骤正是咔唑环的 合成, 路线如 Scheme 18 所示.

2008 年, Mohanakrishnan 小组 ${ }^{[44]}$ 以化合物 50 和多种 取代苯为原料, 通过 Domino 电环化反应, 经 $\mathrm{ZnBr}_{2}$ 催化 一锅法合成了一系列咔唑衍生物, 并得到两个化合物的 晶体结构, 但收率不高, 在 $25 \% \sim 62 \%$ 之间(Scheme 19). 2009 年, 他们 ${ }^{[45]}$ 采用化合物 $\mathbf{5 2}$ 代替 $\mathbf{5 1}$, 合成了一系列 结构更为复杂的咔唑衍生物, 遗憾的是该方法的收率依<smiles>COC(=O)C(=O)C1(CCC(C)=O)CCCN(C(=O)Cc2cn(C)c3c(OC)c(OC)ccc23)C1=O</smiles><smiles></smiles>

$97 \%$

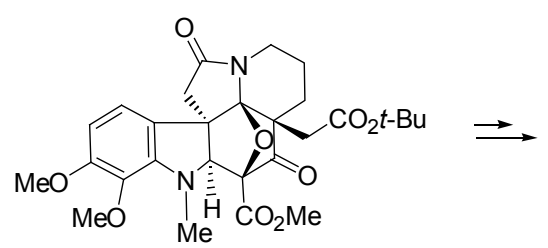<smiles>COc1ccc2c(c1OC)N(C)C1C=CC34CC(=O)CCN3CCC4(C)[C@]21C</smiles>

Scheme 18

然没有提高 $(25 \% \sim 62 \%)$ (Scheme 19). 同年，他们 ${ }^{[46]}$ 又 采用化合物 53 及其类似物为原料，并使用 $\mathrm{DMF} / \mathrm{DMA}$ 作为反应体系, 成功合成了多个胺基取代的咔唑, 收率 较 $\mathrm{ZnBr}_{2}$ 催化方法有所提高，在 $55 \% \sim 73 \%$ 之间(Scheme 19).

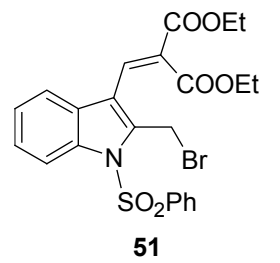

$\operatorname{Ar}^{1} \mathrm{H}$
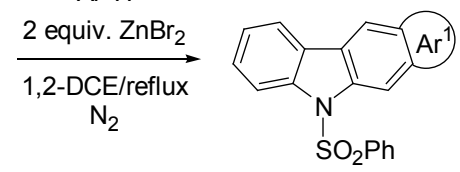

$25 \% \sim 62 \%$<smiles>[R]OC(=O)/C(=C/c1c(CBr)n(S(=O)(=O)c2ccccc2)c2ccc([R])cc12)OCC</smiles>

52<smiles>CCOC(=O)C(=Cc1c(C)n(S(=O)(=O)c2ccccc2)c2ccccc12)C(=O)OCC</smiles>

53

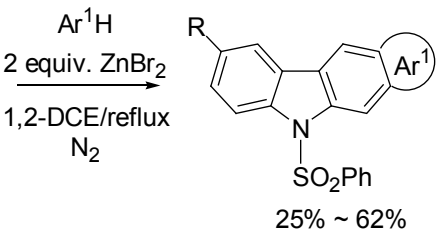

$25 \% \sim 62 \%$<smiles>CCOC(=O)c1ccc2c(c1)c1ccccc1n2-c1ccccc1</smiles>

Scheme 19 
2009 年, $\mathrm{Ma}$ 等 ${ }^{[47]}$ 以 1-(2-吲哚基)-2,3-丁二烯-1-醇为 原料, 尝试使用 $\mathrm{PtCl}_{4}, \mathrm{PtCl}_{2}, \mathrm{AgBF}_{4}, \mathrm{AuCl}\left(\mathrm{PPh}_{3}\right)$ 催化剂 合成4-取代咔唑, 实验结果显示以 $\mathrm{PtCl}_{2}$ 作催化剂, 甲苯 作溶剂效果最好, 收率达到 90\% (Scheme 20). 他们认为 其合理的反应机理中应包含金属卡宾中间体 $\mathrm{E}, \mathrm{PtCl}_{2}$ 中 的 $\mathrm{Pt}$ 原子通过与 $\mathbf{A}$ 的丙二烯单元配位形成中间体 $\mathbf{B}$, 吲 哚 $\mathrm{C}(3)$ 亲核进攻金属活化的 $\mathrm{C}=\mathrm{C}$ 双键，随后羟基发生 质子化脱去 $\mathrm{H}_{2} \mathrm{O}$, 形成烯铂卡宾中间体 $\mathbf{E}$, 最终经过 1,2-H 迁移得到目标产物. 2009 年, Mohanakrishnan 小 组 ${ }^{[88]}$ 采用化合物 $\mathbf{5 4}$ 和烯烃或炔烃为原料, 在 $t-\mathrm{BuOK} /$ THF 体系中, 通过迈克尔加成反应成功合成 1,2,3,4-四 取代咔唑, 但收率仅为 $32 \% \sim 77 \%$ 左右, 而且还有很多 反应根本不能进行, 尽管如此它还是为我们提供了一种 新的咔唑合成手段(Scheme 21).
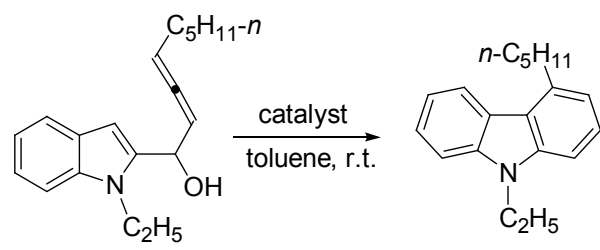

$\mathrm{PtCl}_{4}, 65 \%$

$\mathrm{AgBF}_{4}, 13 \%$

$\mathrm{AuCl}\left(\mathrm{PPh}_{3}\right),<7 \%$

$\mathrm{PtCl}_{2}, 90 \%$

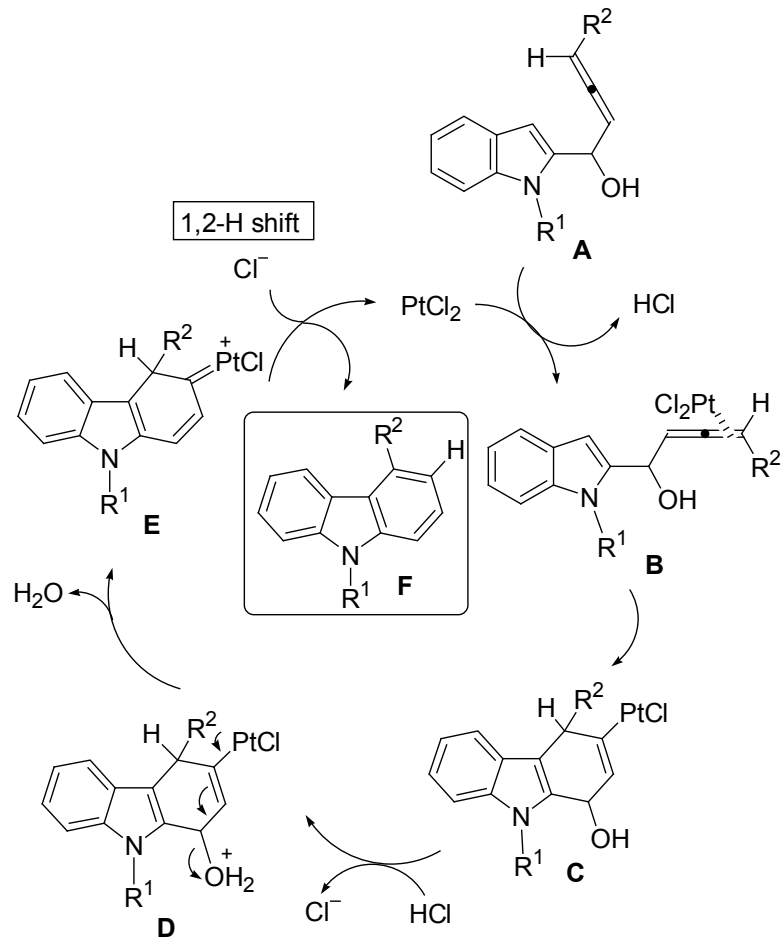

Scheme 20

2010 年, Mal 等 ${ }^{[49]}$ 以吲哚酮为原料, 在 LDA 存在条 件下与马来酸二甲酯反应环合得到咔唑二酯中间体, 其 收率很低, 仅有 $10 \% \sim 15 \%$, 后者经水解、Claisen 重排 等一系列反应可合成得到 clausevatine D (Scheme 22).

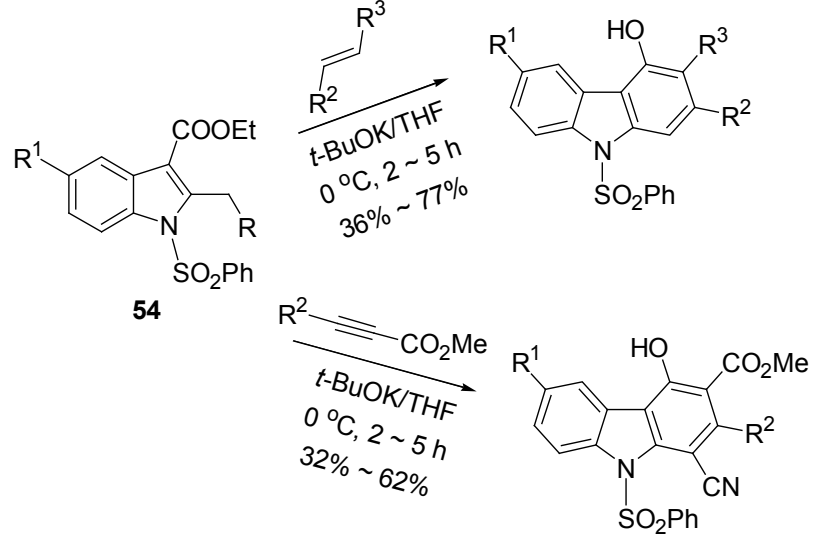

Scheme 21<smiles>CC(C)/C=C/C1OC(=O)c2c1c1ccccc1n2Cc1ccccc1</smiles>

55
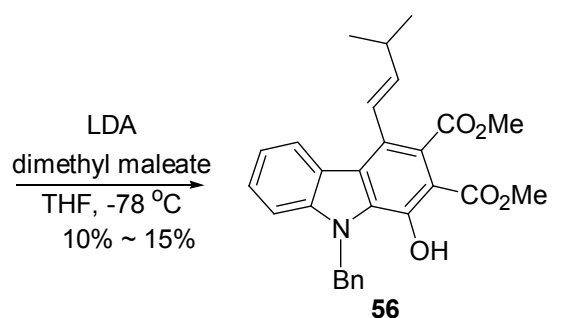

$30 \%$ aq. $\mathrm{KOH}-\mathrm{MeOH}$ reflux, $3 \mathrm{~h}$

$68 \%$

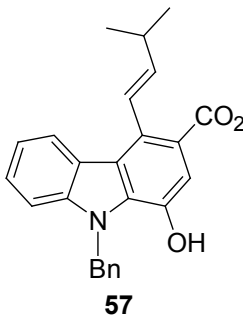

(1) $m$-CPBA

$\mathrm{CO}_{2} \mathrm{H} \quad \mathrm{CH}_{2} \mathrm{Cl}_{2}$ $60 \%$ $\underset{\text { (2) aq. } \mathrm{NaHCO}_{3}}{\stackrel{0}{\circ} \mathrm{C} \sim \text { r.t. }}$

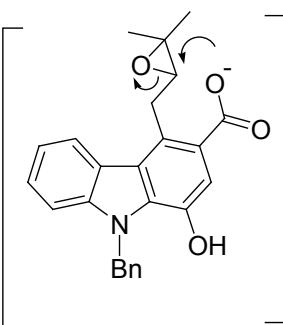

58

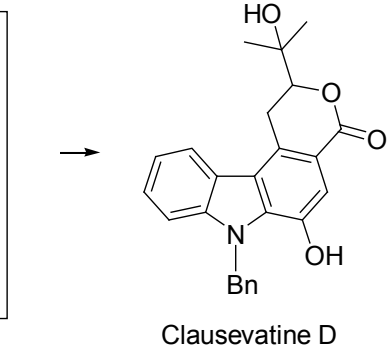

Scheme 22

\section{4 其他方法}

2007 年, Ackermann 等 ${ }^{[50]}$ 报道了一种新型 Pd 催化 Domino $\mathrm{N}-\mathrm{H} / \mathrm{C}-\mathrm{H}$ 键活化反应合成咔唑衍生物的方 法. 该方法以邻二卤(杂)芳烃和苯胺或取代苯胺为原料, 以 $\mathrm{Pd}(\mathrm{OAc})_{2}$ 为催化剂, 成功合成了一系列咔唑化合物, 收率在 57\% 81\%之间(Eq. 23). 
<smiles>[R]Nc1cc[R]([R])cc1</smiles>

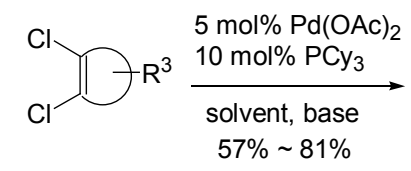<smiles>[R]c1ccc2c(c1)N([R])C1CC([R])CCC21</smiles>

三氟甲磺酸控制的芳香磺胺连续成环方法也可应 用于咔唑的合成, 2007 年, Török 等 ${ }^{[51]}$ 采用此方法成功合 成了 $N$-芳香磺酰基取代的吡咯、吲哚和咔唑. 通过控制 三氟甲磺酸的投料比例, 可以控制反应进行的程度, 从 而控制最终产物. 其中, 该方法合成的 $N$-芳香磺酰基咔 唑的收率高达 75\% 86\% (Scheme 23).

$$
\text { }
$$<smiles>COS(=O)(=O)n1c2ccccc2c2ccccc21</smiles>

\section{$\mathrm{Ar}=\mathrm{C}_{6} \mathrm{H}_{5}, p-\mathrm{CH}_{3} \mathrm{C}_{6} \mathrm{H}_{4}, o-\mathrm{CH}_{3} \mathrm{C}_{6} \mathrm{H}_{4}$,} $p-\mathrm{CH}_{3} \mathrm{OC}_{6} \mathrm{H}_{4}, p-\mathrm{BrC}_{6} \mathrm{H}_{4}, p-\mathrm{NO}_{2} \mathrm{C}_{6} \mathrm{H}_{4}$, $p-\mathrm{ClC}_{6} \mathrm{H}_{4}$, Naphth-2-yl

\section{Scheme 23}

2008 年, Lebold 和 $\mathrm{Kerr}^{[52]}$ 在全合成 Clausamines $\mathrm{A} \sim \mathrm{D}$ 的过程中, 将苯醌亚胺和 1-乙烯基环已烯通过 Diels-Alder 反应得到关键中间体二氢萗化合物, 经过双 键的氧化裂解, 得到的二羰基化合物经酸化处理得到取 代的四氢咔唑, 再进行脱氢反应即可得到取代咔唑. 该 方法三步反应总收率高达 $95 \%$, 适用于合成特定取代位 置的咔唑化合物(Scheme 24).

2008 年, Knölker 小组 ${ }^{[53]}$ 首次全合成了全系列的 antiostatins A 和 B. 合成过程中, 芳胺 59 与铁配合物盐 60 亲电取代反应生成铁配合物 61 , 在过量的 $\mathrm{Cp}_{2} \mathrm{FePF}_{6}$ 和碳酸钠存在条件下, 再经氧化环化得到咔唑化合物 62 (Scheme 25).

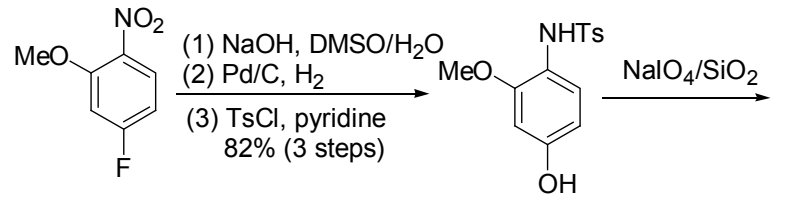<smiles>C=CC1=CCCCC1</smiles>

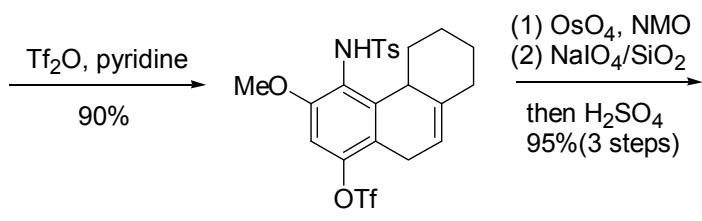<smiles></smiles>

Scheme 24<smiles>[R]Cc1c(N)ccc(OC)c1C</smiles><smiles>[R]Cc1c(C)c(OC)cc([C@H]2C=C(C=O)C=CC2)c1N</smiles><smiles>[R2]CCc1c(C)c(OC)cc2c1[nH]c1ccccc12</smiles>

(a) $\mathrm{CH}_{3} \mathrm{CN}, 82{ }^{\circ} \mathrm{C}, 1 \sim 2 \mathrm{~h}$; (b) $\mathrm{Cp}_{2} \mathrm{FePF}_{6}, \mathrm{Na}_{2} \mathrm{CO}_{3}, \mathrm{CH}_{2} \mathrm{Cl}_{2}$, r.t., $3 \sim 5$ d.

Scheme 25

2009 年, Eisch 等 ${ }^{[54]}$ 报道了 6,7-二苯基二苯并 $[e, g][1,4]$ 二氮杂环辛四烯的重排反应，在 $\mathrm{Na} / \mathrm{THF}$ 条件 下, 重排生成 $N$-(2-胺基-1,2-苯乙烯基)咔唑. 该重排反 应为我们合成特定的咔唑衍生物提供了一条新的途径 (Scheme 26).

2010 年, Hayashi 等 ${ }^{[55]}$ 报道了以四氢咔唑通过活性 $\mathrm{Pd} / \mathrm{C}$-乙烯体系或 $\mathrm{C}-\mathrm{O}_{2}$ 体系脱氢制备咔唑衍生物的方 法. 研究发现, 两种体系均可方便合成得到咔唑及其 


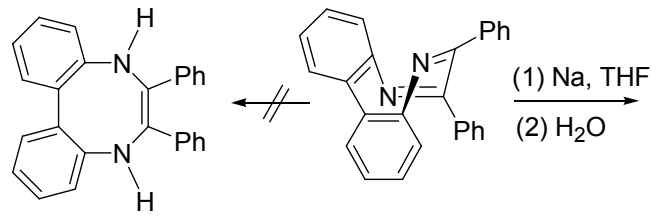<smiles>NC(=C(c1ccccc1)c1ccccc1)n1c2ccccc2c2ccccc21</smiles>

$Z$ and $E$ isomers

Scheme 26

衍生物, 其中 Pd/C-乙烯体系的收率要高于后者(Eq. 24).
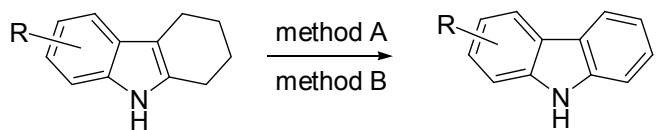

Method A: Pd/C-ethylene; method B: activated carbon- $\mathrm{O}_{2}$

2011 年 Knölkr 等 ${ }^{[56]}$ 报道了铁配合物中间体诱导分 子内氧化环合反应制备咔唑衍生物的方法. 其利用合成 chrestifoline 的成功经验, 以三崖基铁-环已二烯配合物 为起始原料先与氨基苯并吡喃反应得到关键铁配合物 中间体, 然后在碘、吡啶存在条件下通过铁诱导分子内 氧化环合反应成功合成得到咔唑并吡喃衍生物, 收率> $60 \%$ (Scheme 27). 该方法原料易得且操作简便，遗憾的 是在环合步骤中由于对环已二烯端 $\mathrm{C}-\mathrm{C}$ 键的 $\alpha$ 位没有 选择性，因而该方法仅能制备复杂的多取代咔唑衍生 物.

\section{5 结论与展望}

咔唑因其独特的结构及理化性质日益引起科研人 员的浓厚兴趣, 特别是近年来新型单、多取代咔唑衍生 物被发现具有良好的抗肿瘤及抗惊厥活性, 显示了广阔 的应用前景. 由此, 咔唑及其衍生物的需求量日益增长. 传统咔唑制备方法如 Borsche 合成法等虽然具有操作简 便、成本低廉的优点，但其仅能合成个别简单的咔唑衍 生物, 而无法胜任复杂咔唑衍生物特别是特定位点、特 定取代基团的咔唑衍生物的合成任务. 因此, 开发新型 咔唑及其衍生物的合成方法十分有益.

催化科学的发展极大地促进了咔唑及其衍生物合 成方法发展，贵金属和过渡金属配合物催化剂的应用极 大地丰富了咔唑及其衍生物的合成手段. 特别是 Suzuki 偶联反应、Domino 电环化反应以及分子内氧化偶联反

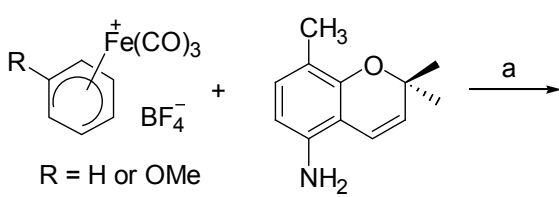

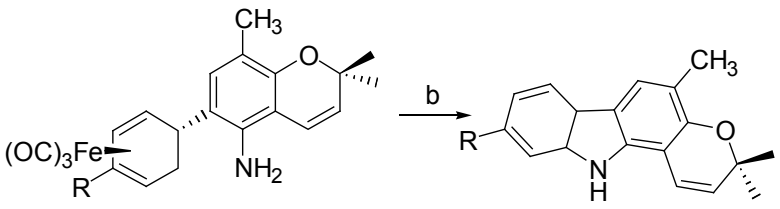

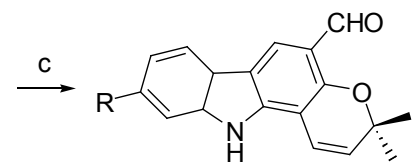

(a) $\mathrm{MeCN}$, r.t. $\mathrm{R}=\mathrm{H}: 94 \%, \mathrm{R}=\mathrm{OMe}: 95 \%$

(b) iodine, pyridine, $90^{\circ} \mathrm{C}, 6 \mathrm{~h}$. $\mathrm{R}=\mathrm{H}: 61 \%$, R = OMe: $63 \%$

(c) $\mathrm{DDQ}, \mathrm{MeOH}-\mathrm{THF}-\mathrm{H}_{2} \mathrm{O}(3: 1: 1)$, r.t., $90 \mathrm{~min} . \mathrm{R}=\mathrm{H}: 96 \%$, $\mathrm{R}=\mathrm{OMe}: 93 \%$

\section{Scheme 27}

应等使得复杂咔唑衍生物的合成日益简便，大规模制备 复杂咔唑衍生物也由理论逐渐变为现实. 相信随着研究 的不断深入，咔唑及其衍生物的应用范围被不断拓宽， 各种新的合成方法还会不断涌现. 科学是无止境的, 咔 唑及其衍生物的合成研究远未结束, 仍有待进一步丰富 和发展。

\section{References}

[1] (a) Graebe, C.; Ullmann, F. Ann. 1896, 291, 16 (b) Drechsel, E. J. Prakt. Chem. 1858, 38, 69.

[2] Preston, R. W. G.; Tucker, S. H.; Cameron, J. M. L. J. Chem. Soc. 1942, 500.

[3] (a) Bucherer, H. T. J. Prakt. Chem. 1904, 69, 49. (b) Borsche, W.; Feise, M. Ber. Dtsch. Chem. Ges. 1904, 20, 378.

[4] Yu, M.-J.; Chen, Z.-J; Lu, D.-D. Dyestuff Ind. 1998, 35, 21 (in Chinese).

(俞马金，陈中峻，陆大东，染料工业, 1998, 35, 21.)

[5] Chen, M.-W.; Gan, L.-Z. Organic Heterocyclic Compounds, Higher Education Press, Beijing, 1990, p. 56 (in Chinese).

(陈敏为; 甘礼骓，有机杂环化合物，高等教育出版社，北京， 1990, p. 56.)

[6] Sanz, R.; Escribano, J.; Pedrosa, M. R.; Aguado, R.; Arnaiz, F. J. Adv. Synth. Catal. 2007, 349, 713.

[7] Naffziger, M. R.; Ashburn, B. O.; Perkins, J. R.; Carter, R. G. J. Org. Chem. 2007, 72, 9857.

[8] Creencia, E. C.; Kosaka, M.; Muramatsu, T.; Kobayashi, M.; Iizuka, T.; Horaguchi, T. J. Heterocycl. Chem. 2009, 46, 1309.

[9] Pudlo, M.; Csanyi, D.; Moreau, F.; Hajos, G.; Riedl, Z.; Sapi, J. Tetrahedron 2007, 63, 10320.

[10] Stokes, B. J.; Jovanovic, B.; Dong, H. J.; Richert, K. J.; Riell, R. D.; Driver, T. G. J. Org. Chem. 2009, 74, 3225.

[11] Shou, W. G.; Li, J. A.; Guo, T. X.; Lin, Z. Y.; Jia, G. C. Organometallics 2009, 28, 6847. 
[12] Jean, D. J. S.; Poon, S. F.; Schwarzbach, J. L. Org. Lett. 2007, 9, 4893.

[13] Jordan-Hore, J. A.; Johansson, C. C. C.; Gulias, M.; Beck, E. M.; Gaunt, M. J. J. Am. Chem. Soc. 2008, 130, 16184.

[14] Tsang, W. C. P.; Munday, R. H.; Brasche, G.; Zheng, N.; Buchwald, S. L. J. Org. Chem. 2008, 73, 7603.

[15] Li, B. H.; Tian, S. L.; Fang, Z.; Shi, Z. H. Angew. Chem. Int. Ed. 2008, 47, 1115.

[16] Kajiyama, D.; Inoue, K.; Ishikawa, Y.; Nishiyama, S. Tetrahedron 2010, 66, 9779.

[17] Cho, S. H.; Yoon, J.; Chang, S. J. Am. Chem. Soc. 2011, 133, 5996.

[18] Ueno, A.; Kitawaki, T.; Chida, N. Org. Lett. 2008, 10, 1999.

[19] Li, E. D.; Xu, X. B.; Li, H. F.; Zhang, H. M.; Xu, X. L.; Yuan, X. Y.; Li, Y. Z. Tetrahedron 2009, 65, 8961.

[20] Kawaguchi, K.; Nakano, K.; Nozaki, K. Org. Lett. 2008, 10 (6), 1199.

[21] Watanabe, T.; Ueda, S.; Inuki, S.; Oishi, S.; Fujii, N.; Ohno, H. Chem. Commun. 2007, 43, 4516.

[22] Watanabe, T.; Oishi, S.; Fujii, N.; Ohno, H. J. Org. Chem. 2009, 74, 4720 .

[23] Liegault, B.; Lee, D.; Huestis, M. P.; Stuart, D. R.; Fagnou, K. J. Org. Chem. 2008, 73, 5022.

[24] Sridharan, V.; Martin, M. A.; Menéndez, J. C. Eur. J. Org. Chem. 2009, 27, 4614.

[25] Tobisu, M.; Kita, Y.; Ano, Y.; Chatani, N. J. Am. Chem. Soc. 2008, 130, 15982 .

[26] Bedford, R. B.; Butts, C. P.; Haddow, M. F.; Osborne, R.; Sankey, R. F. Chem. Commun. 2009, 32, 4832.

[27] Shimizu, M.; Hirano, K.; Satoh, T.; Miura, M. J. Org. Chem. 2009, 74, 3478 .

[28] Buden, M. E.; Vaillard, V. A.; Martin, S. E.; Rossi, R. A. J. Org. Chem. 2009, 74, 4490.

[29] Tsvelikhovsky, D.; Buchwald, S. L. J. Am. Chem. Soc. 2010, 132, 14048.

[30] Abbiati, G.; Canevari, V.; Facoetti, D.; Rossi, E. Eur. J. Org. Chem. 2007, 2007, 517.

[31] Gioia, C.; Hauville, A.; Bernardi, L.; Fini, F.; Ricci, A. Angew. Chem. Int. Ed. 2008, 47, 9236

[32] Tao, M.; Park, C. H.; Josef, K.; Hudkins, R. L. J. Heterocycl. Chem. 2009, 46, 1185.

[33] Martinez-Esperon, A. F.; Rodriguez, D.; Castedo, L.; Sáa, C. Tetrahedron 2008, 64, 3674.
[34] Alayrac, C.; Schollmeyer, D.; Witulski, B. Chem. Commun. 2009, 1464.

[35] Tsuchimoto, T.; Matsubayashi, H.; Kaneko, M.; Nagase, Y.; Miyamura, T.; Shirakawa, E. J. Am. Chem. Soc. 2008, 130, 15823.

[36] Bourderioux, A.; Kassis, P.; Merour, J. Y.; Routier, S. Tetrahedron 2008, 64, 11012.

[37] Yamashita, M.; Hirano, K.; Satoh, T.; Miura, M. Org. Lett. 2009, 11, 2337.

[38] Yamashita, M.; Horiguchi, H.; Hirano, K.; Satoh, T.; Miura, M. J. Org. Chem. 2009, 74, 7481.

[39] Wang, C. L.; Zhang, W.; Lu, S. C.; Wu, J. F.; Shi, Z. J. Chem. Commun. 2008, 41, 5176.

[40] Tiano, M.; Belmont, P. J. Org. Chem. 2008, 73, 4101.

[41] acoetti, D.; Abbiati, G.; Rossi, E. Eur. J. Org. Chem. 2009, 17, 2872.

[42] adav, A. K.; Peruncheralathan, S.; Ila, H.; Junjappa, H. J. Org. Chem. 2007, 72, 1388.

[43] Hong, X.; France, S.; Padwa, A. Tetrahedron, 2007, 63, 5962.

[44] Mohanakrishnan, A. K.; Dhayalan, V.; Clement, J. A.; Balamurugan, R.; Sureshbabu, R.; Kumar, N. S. Tetrahedron Lett. 2008, 49, 5850.

[45] Dhayalan, V.; Clement, J. A.; Jagan, R.; Mohanakrishnan, A. K. Eur. J. Org. Chem. 2009, 4, 531.

[46] Sureshbabu, R.; Balamurugan, R.; Mohanakrishnan, A. K. Tetrahedron 2009, 65, 3582.

[47] Kong, W. Q.; Fu, C. L.; Ma, S. M. Chem. Commun. 2009, 30, 4572.

[48] Ramesh, N.; Rajeshwaran, G. G.; Mohanakrishnan, A. K. Tetrahedron 2009, 65, 3592.

[49] Jana, A. K.; Mal, D. Chem. Commun. 2010, 46, 4411.

[50] Ackermann, L.; Althammer, A. Angew. Chem., Int. Ed. 2007, 46, 1627.

[51] Abid, M.; Teixeira, L.; Török, B. Tetrahedron Lett. 2007, 48, 4047.

[52] Lebold, T. P.; Kerr, M. A. Org. Lett. 2008, 10, 997.

[53] Knott, K. E.; Auschill, S.; Jager, A.; Knölker, H. J. Chem. Commun. 2009, $12,1467$.

[54] Eisch, J. J.; Manchanayakage, R. N.; Rheingold, A. L. Org. Lett. 2009, 11, 4060.

[55] Tanaka, T.; Okunaga, K.; Hayashi, M. Tetrahedron Lett. 2010, 51, 4633.

[56] Gruner, K. K.; Hopfmann, T.; Matsumoto, K.; Jager, A.; Katsuki, T.; Knölker, H. J. Org. Biomol. Chem. 2011, 9, 2057. 